\title{
Multi-scale hydro-morphodynamic modelling using mesh movement methods
}

\author{
Mariana C A Clare · Joseph G Wallwork · Stephan C Kramer · Hilary Weller · Colin J Cotter · Matthew \\ D Piggott
}

This manuscript is a non-peer reviewed EarthArXiv preprint that has been submitted for publication in International Journal in Geomathematics

\begin{abstract}
Hydro-morphodynamic modelling is an important tool that can be used in the protection of coastal zones. The models can be required to resolve spatial scales ranging from sub-metre to hundreds of kilometres and are computationally expensive. In this work, we apply mesh movement methods to a depth-averaged hydro-morphodynamic model for the first time, in order to tackle both these issues. Mesh movement methods are particularly well-suited to coastal problems as they allow the mesh to move in response to evolving flow and morphology structures. This new capability is demonstrated using test cases that exhibit complex evolving bathymetries and have moving wet-dry interfaces. In order to be able to simulate sediment transport in wet-dry domains, a new conservative discretisation approach has been developed as part of this work, as well as a sediment slide mechanism. For all test cases, we demonstrate how mesh movement methods can be used to reduce discretisation error and computational cost. We also show that the optimal parameter choices in the mesh movement monitor functions are fairly predictable based upon the physical characteristics of the test case, facilitating the use of mesh movement methods on further problems.
\end{abstract}

Keywords mesh adaptation; mesh movement; sediment transport; morphology

\section{Introduction}

Over the last few decades, hydro-morphodynamic models have been increasingly used to simulate erosion in both fluvial and coastal zones (see Amoudry and Souza (2011); Papanicolaou et al. (2008)). Unfortunately, these models are generally computationally expensive, especially as they are often required to simulate very long-term morphological effects with relatively small timesteps in order to resolve hydrodynamic features such as waves and tides. These models must also often run multiple times for calibration purposes and to quantify uncertainty, as for example in Harris et al. (2018), Callaghan et al. (2013) and Villaret et al. (2016), thus further increasing the computational cost. Therefore, many fluvial and coastal scenarios are infeasible to model using standard fixed meshes of appropriate resolution. In addition, when applied to coastal regions, hydro-morphodynamic models must resolve problems with complex and fundamentally multi-scale domains. Unstructured mesh models, such as those based on finite element methods (Piggott et al., 2008a,b), are ideally suited to providing the required mesh flexibility, and multiple tools exist for generating multi-scale fixed meshes which are suitable for a wide range of geophysical applications (such as Avdis et al. (2018)). However, these are insufficient in cases with significant morphology changes as the areas which require finer mesh resolution vary throughout the simulation. A solution to this time-dependent multi-scale issue is to use mesh adaptation methods, such as mesh movement and $h$-refinement, which offer the potential to improve result accuracy and/or reduce computational cost.

The focus and main novelty of this work is that we apply mesh movement methods (also known as $r$-adaptation) (see Budd et al. (2009)) to hydro-morphodynamic models. For these methods, the mesh topology is fixed and the (fixed

M C A Clare

Imperial College London, U.K.

E-mail: m.clare17@imperial.ac.uk

J G Wallwork; S C Kramer; C J Cotter; M D Piggott

Imperial College London, U.K.

H Weller

University of Reading, U.K. 


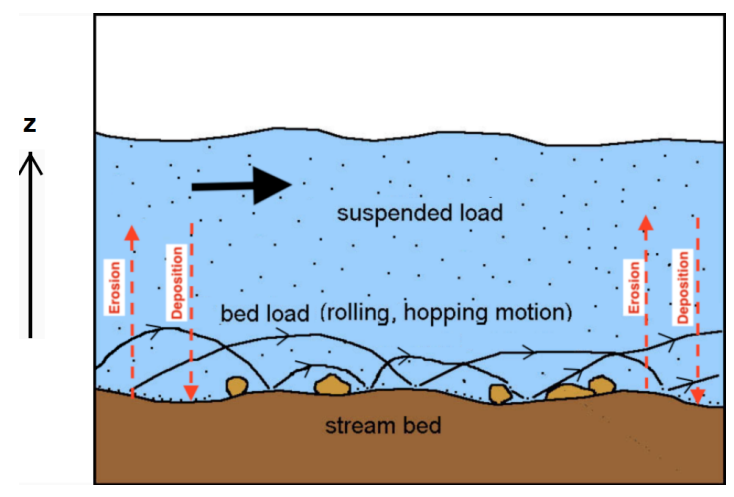

Fig. 1 Diagram of sediment transport processes. Adapted from Geology Cafe (2015)

number of) mesh nodes are dynamically moved, thus allowing different regions of the domain to vary between low and 20 high resolution as flow structures pass through them. Previous works applying mesh adaptation to hydro-morphodynamic models, for example Mayne et al. (2002); Delandmeter (2017); Benkhaldoun et al. (2013), have used $h$-refinement, where the mesh topology is more substantially altered with cells locally created or destroyed and mesh connectivity altered (Piggott et al., 2009, 2006). Whether mesh movement methods or $r$-adaptation are more appropriate is dependent on the case considered. In most hydro-morphodynamic problems, regions warranting the highest mesh resolution move 25 continuously with the flow, meaning that mesh movement is arguably more appropriate than $h$-refinement since it can track the features of interest. It should be noted though, that if features were to suddenly appear and extra degrees of freedom be required, then $h$-refinement, or combined mesh movement with $h$-refinement, would be more appropriate. Other advantages of mesh movement are that it avoids the problem of hanging nodes which must be addressed with $h$-refinement, and especially adaptive mesh refinement (AMR) techniques; and that the number of mesh nodes remain fixed during the simulation, thus simplifying data structures.

In this work, we use the 2D depth-averaged coupled hydro-morphodynamic model of Clare et al. (2021b) to simulate erosion and deposition, which models both suspended sediment transport and bedload transport for non-cohesive sediment with bed updates (see Figure 1). Depth-averaged models have the advantage of being computationally cheaper than a full 3D model and yet, unlike 2D-vertical models, are still able to capture variations in the cross-stream directions,

35 as well as changes to the bed. Our model uses a discontinuous Galerkin finite element discretisation on an unstructured mesh, which has several advantages including being locally mass conservative, meaning sediment is conserved on an element-by-element level, which is an advantage when coupling between the hydrodynamic and morphodynamic model (Dawson et al., 2004); being well-suited to advection-dominated problems (Kärnä et al., 2017); and being geometrically flexible. Furthermore, our model has key advantages and/or differences to the hydro-morphodynamic models for which $h$-refinement has been applied: it is a depth-averaged model able to capture variations in cross-stream directions (Mayne et al. (2002) uses a 2D-vertical model which is unable to do this); it simulates bedlevel changes meaning erosion and deposition can be modelled (Delandmeter (2017) does not have this capability), and uses finite element methods (Benkhaldoun et al. (2013) uses finite volume methods).

Our hydro-morphodynamic model is developed within the finite element coastal ocean modelling system Thetis 45 (Kärnä et al., 2017) built using the Firedrake Python-based code generation PDE solver framework (Rathgeber et al., 2016)). This framework is well suited for the inclusion of $r$-adaptation methods since these often require the solution of additional complex non-linear PDEs, which Firedrake is designed to make readily solvable (see McManus et al. (2017)). In addition, we take advantage of the adjoint framework within Firedrake to calibrate values of uncertain parameters in our model (see Farrell et al. (2013)). Mesh adaptation has already been implemented in the wider Firedrake framework,

50 for example in McManus et al. (2017); McRae et al. (2018); Barral et al. (2016); Wallwork et al. (2020a). Integration of the mesh data structures with the underlying PETSc representation (Lange et al., 2016) has laid the groundwork for the use of anisotropic metric-based methods in Firedrake (Barral et al., 2016). This was used to apply mesh adaptation methods to the hydrodynamic component of Thetis' 2D model for the first time in Wallwork et al. (2020b). The present work is the first to use mesh adaptation with a coupled model in the Firedrake framework and the first to use mesh movement methods in Thetis.

We test our novel hydro-morphodynamic model mesh-movement framework using multi-scale test cases with complex evolving bathymetries which are difficult to capture accurately on coarse and/or fixed meshes, that may incorrectly represent small structures. We also consider test cases with a moving wet-dry interface that can be difficult to capture on a fixed mesh, a frequent scenario in the modelling of coastal zones. In order to be able to simulate sediment transport

${ }_{60}$ in wet-dry domains, a new conservative discretisation approach has been developed as part of this work, as well as a sediment slide mechanism. Hence, we are able to establish a generalised mesh movement methodology that can be applied to a variety of fluvial and coastal zone test cases. 
The remainder of this paper is structured as follows: in Section 2 we outline the mesh movement methods used; in Section 3 we give a brief outline of the hydro-morphodynamic model including the new conservative discretisation approach; in Section 4 we apply our mesh movement methods to a series of test cases and finally in Section 5 we conclude this work.

\section{Mesh Movement}

There are a variety of approaches that can be taken to apply mesh movement. These include (but are not limited to): imposing a prescribed mesh velocity (Donea et al., 2017); re-interpreting the mesh as a structure of stiff beams (Farhat et al., 1998); and enforcing mesh transformations using monitor functions (Huang et al., 1994; Budd and Williams, 2009).

In this work, we consider the monitor function based approach, for which the 'physical' mesh - upon which the prognostic equations are solved - is moved during the time period of simulation, with its 'density' prescribed by a userprovided monitor function. The physical mesh is moved by defining it to be the image of some mapping applied to a fixed 75 reference mesh, which determines the way in which the mesh is moved. The monitor function concept was first proposed in White (1979). In that work, in the context of a one dimensional PDE, a monitor function based on the arc length of the PDE solution was used. The monitor-based framework enforces the even distribution of the monitor function across mesh elements, meaning that regions with high values have elements with small areas and vice versa. This is typically achieved through the solution of an auxiliary PDE which describes the mesh movement (such as Huang et al. (1994);

80 Budd and Williams (2009)). As may be expected, the choice of monitor function greatly impacts the geometry of the moved mesh and should therefore be chosen with care.

Both the PDE prescribing the mesh movement and the monitor functions are considered to be time-dependent, meaning that the physical mesh changes with time. As mentioned in Section 1, interpolating between meshes during the PDE time integration loop can be computationally expensive and introduces additional errors. For this reason, we could 85 formulate the PDE and mesh movement equation as a single system of equations to be solved at each timestep as in an Arbitrary Lagrangian-Eulerian (ALE) type approach (Budd and Williams, 2009; Donea et al., 2017). This would remove the need for mesh-to-mesh interpolation (Piggott et al., 2006), but it is not obvious that these model improvements are worth the cost associated with being constrained to move the mesh at every timestep. As such, implementation and numerical experimentation for ALE methods within the context of hydro-morphodynamic models remains the topic of

90 future work. Instead, in this work fields are transferred between meshes using conservative Galerkin projection, achieved using libsupermesh (Maddison et al., 2017).

\subsection{Equidistribution and the Monge-Ampère equation}

The approach to mesh movement used in this work is concerned with obtaining a discrete representation of a sufficiently smooth map,

$$
\mathbf{x}: \Omega_{C} \times[0, T] \rightarrow \Omega_{P}, \quad \mathbf{x}\left(\mathscr{H}_{C}, t\right)=\mathscr{H}_{P}(t), \quad t \in[0, T] .
$$

Here $\Omega_{C} \subset \mathbb{R}^{n}$ is a computational reference domain, which remains fixed and $\mathscr{H}_{C}$ is an associated computational mesh. The physical domain is denoted by $\Omega_{P}:[0, T] \rightarrow \mathbb{R}^{n}$, is allowed to vary with time and has an associated physical mesh, $95 \mathscr{H}_{P}$, upon which our hydro-morphodynamic model is solved. As with the domains, $\mathscr{H}_{P}=\mathscr{H}_{P}(t)$ is allowed to vary with time, whereas $\mathscr{H}_{C}$ remains fixed.

The map (1) has certain constraints imposed on it. In particular, the user-specified monitor function, $m: \Omega_{P} \times[0, T] \rightarrow$ $(0, \infty)$ must be equidistributed, in the sense that

$$
m \operatorname{det} \underline{\mathbf{J}}=\theta \quad \text { where } \quad \underline{\mathbf{J}}=\frac{\partial \mathbf{x}}{\partial \xi}
$$

is the Jacobian transform with respect to the computational coordinate $\xi \in \Omega_{C}$. The normalisation coefficient $\theta:[0, T] \rightarrow$ $(0, \infty)$ acts to conserve the domain volume. In this work, we do not consider boundary deformations and hence will always assume that $\Omega_{P}=\Omega_{C}=: \Omega$ meaning $\theta$ is a constant. The mesh movement problem can thus be stated as follows: for a given $m$ and $\theta$, find a map, $\mathbf{x}$, which satisfies (2).

In dimension $n>1$ the mesh movement problem is ill-posed, meaning additional constraints must be imposed on the map (1). Our hydro-morphodynamic model described in Section 3 is in $n=2$ spatial dimensions and therefore this is indeed the case in this work. Following the optimal transport approach advocated in McRae et al. (2018); Budd and Williams (2009); Budd et al. (2013); Weller et al. (2016), we assume that the map takes the form

$$
\mathbf{x}(\xi)=\xi+\nabla \phi_{\xi}(\xi)
$$


for some scalar potential $\phi: \Omega_{C} \times[0, T] \rightarrow \Omega_{P}$. This form of map prevents mesh tangling (where one or more mesh elements become inverted): that its curl is zero implies no element orientation changes occur. (Assuming a sufficiently accurate solution of (2).) Substituting (3) into (2) yields

$$
m \operatorname{det}(\underline{\mathbf{I}}+\underline{\mathbf{H}}(\phi))=\theta
$$

where $\underline{\mathbf{I}}$ is the identity matrix in $\mathbb{R}^{n \times n}$ and $\underline{\mathbf{H}}(\phi)$ denotes the Hessian of the potential with respect to the computational coordinates. Equation (M-A) is a nonlinear PDE of Monge-Ampère type.

Under an appropriate choice of monitor function, the elliptic PDE (M-A) has been shown to be capable of producing equidistributed meshes which admit more accurate solutions of the underlying PDE (in this case the hydromorphodynamic model) without increasing the number of degrees of freedom or modifying the mesh topology (for example, see McRae et al. (2018); Budd and Williams (2009); Budd et al. (2013); Weller et al. (2016)).

A criticism of mesh movement methods which are driven by solutions of Monge-Ampère type equations is that such equations require a degree of convexity of domain geometry which are not always present in ocean domains bounded by coasts (see Theorem 4.3 of Budd and Williams (2009)). However, for many realistic coastlines, the use of wetting-and-drying scheme in the hydrodynamics solver such as the one present in our model (see Section 3) means that domain convexity can be ensured, because the domain boundary is no longer constrained to topographic contours and may be chosen as a convex superset of the domain. Moreover, convexity is a sufficient but not necessary condition for solveability and problems with non-convex domains can often be solved in practice.

\subsection{Implementing mesh movement}

115 As discussed above, in order to implement mesh movement based on (M-A), the user must choose a computational mesh $\mathscr{H}_{C}$, an initial physical mesh $\mathscr{H}_{P}$ with an identical topology (usually chosen to coincide with $\mathscr{H}_{C}$ ), a normalisation coefficient $\theta$ and a monitor function $m$. The choice of monitor function is particularly important (see Section 4) because it determines the way in which mesh resolution is to be distributed over the domain.

The mesh movement itself is achieved in an iterative fashion. Each iteration involves the numerical solution of (MA) for the scalar potential on the current physical mesh, followed by the transformation of this mesh according to (3). Equation (M-A) is an elliptic PDE, with two sources of nonlinearity: one from the determinant and another from the product with the monitor function, via the dependence of the physical coordinates on the computational coordinates. Thus, we follow (Budd and Williams, 2009) and (McRae et al., 2018, Section 2.3), parabolising the Monge-Ampère equation

$$
-\frac{\partial}{\partial \tau} \Delta \phi=m \operatorname{det}(\underline{\mathbf{I}}+\underline{\mathbf{H}}(\phi))-\theta
$$

where $\tau$ denotes 'pseudotime' and $\Delta \tau>0$ is a 'pseudotimestep'. As $\phi$ approaches a steady state, the derivative term on the left-hand side converges to zero, whereby the solution of (4) tends to the solution of the elliptic form (M-A). It is proved in Awanou (2015) that the sequence of solutions given by solving (4) converges to the solution of (MA), provided the pseudotimestep is sufficiently small and the initial guess for $\phi$ is sufficiently close to the solution. A pseudotimestep of $\Delta \tau=0.1$ is found to be sufficient in this work. Note, the problems considered in McRae et al. (2018) have periodic boundary conditions, whereas in this work, we consider non-periodic domains. Given that we do not consider boundary deformations, mesh movement within boundary segments is constrained to tangential directions.

To solve (4), still following McRae et al. (2018), we use a mixed finite element method. At pseudotimestep $k$, the forward Euler scheme is applied as follows:

$$
\left\langle\nabla \psi, \nabla \phi_{k+1}\right\rangle_{\mathscr{L}_{2}(\Omega)}=\left\langle\nabla \psi, \nabla \phi_{k}\right\rangle_{\mathscr{L}_{2}(\Omega)}+\Delta \tau\left\langle\psi, m \operatorname{det}\left(\underline{\mathbf{I}}^{+} \underline{\mathbf{H}}_{k}\right)-\theta\right\rangle_{\mathscr{L}_{2}(\Omega)}, \quad \forall \psi \in \Psi,
$$

where $\Psi$ is a scalar function space in which the potential is approximated and $\Sigma$ is a tensor function space in which its Hessian is approximated. The Hessian $\underline{\mathbf{H}}_{k}$ is initialised to zero for the first iteration (assuming that the initial physical mesh coincides with the computational mesh). It is then recovered via an $\mathscr{L}_{2}$ projection in subsequent iterations (Lakkis and Pryer, 2013):

$$
\left\langle\underline{\sigma}, \underline{\mathbf{H}}_{k+1}\right\rangle_{\mathscr{L}_{2}(\Omega)}=-\left\langle\nabla \cdot \underline{\sigma}, \nabla \phi_{k+1}\right\rangle_{\mathscr{L}_{2}(\Omega)}+\left\langle\underline{\sigma} \cdot \widehat{\mathbf{n}}, \nabla \phi_{k+1}\right\rangle_{\mathscr{L}_{2}(\partial \Omega)}, \quad \forall \underline{\sigma} \in \Sigma .
$$

Solving (4) equation repeatedly can be computationally expensive, because each solve involves establishing a new map from $\Omega_{C}$ to $\Omega_{P}$. However, in this work, we employ several strategies to reduce this cost. Firstly, we use the final value of $\phi$ computed during one iteration as the initial guess for the next. This is an appropriate choice provided that the hydro-morphodynamics have not changed too significantly between the iterations. In fact, in Section 4 , we see that due to the slow nature of morphodynamic changes, this is often the case and it can even be excessive to apply mesh movement at every timestep, especially if the modifications to mesh coordinates are only minor. Thus, reduction of the mesh movement frequency provides another method for reducing computational cost. Finally, whilst the exact equidistribution provided by (M-A) is mathematically attractive, it is often unnecessary in practice. Figure 2 shows an 


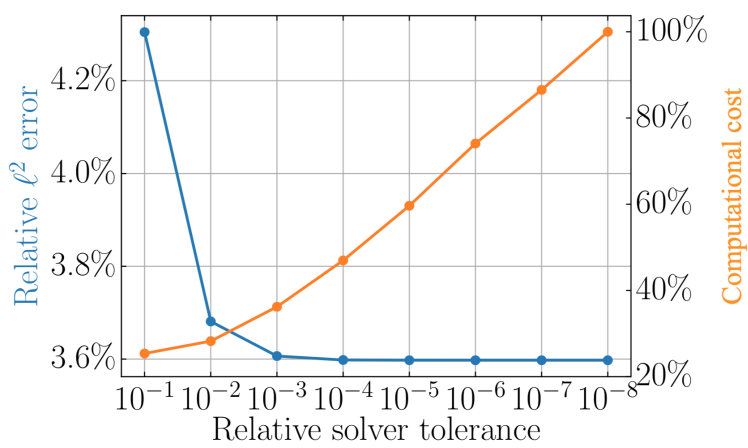

Fig. 2 An example of the trade-off between discretisation error and computational cost with relative solver tolerance for the migrating trench test case. The monitor function (22) has been applied to a mesh with 10 elements in the $x$-direction in the case $\alpha=2, \beta=0$. Error and times are shown as a percentage relative to the fixed mesh case with 10 mesh elements

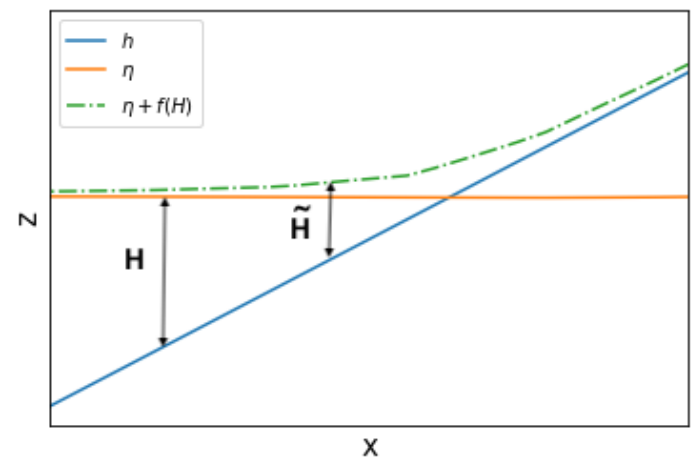

Fig. 3 Wetting and drying scheme diagram showing the relationship between the total water depth $H$ and its modified form $\tilde{H}$

example of the trade-off between discretisation error and computational cost with respect to the relative solver tolerance used to solve (4). It shows that tolerance values above $\mathscr{O}\left(10^{-3}\right)$ result in large gains in computational cost for almost no accuracy benefits. The case in this figure is for the migrating trench test case from Section 4.1 with a mesh size of 10 mesh elements in the $x$-direction. Similar patterns were observed for other numbers of mesh elements and thus throughout this work we use a relative solver tolerance of $10^{-3}$ to solve the parabolised form (4).

\section{Hydro-Morphodynamic Model}

Before applying our mesh-movement hydro-morphodynamic framework to some fluvial and coastal zone test cases, we must describe and develop the hydro-morphodynamic model. In this work, we use the hydro-morphodynamic model derived in Clare et al. (2021b). This model is able to simulate both suspended sediment and bedload transport taking into account gravitational and helical flow effects. To maximise stability, the time derivatives of our model equations are approximated using a fully-implicit backward Euler timestepping scheme. Full details of the model and its development are provided in Clare et al. (2021b).

So far this model has been used for test cases in which the whole domain is wet. However, in coastal problems, there is often a wet-dry interface which must be simulated. We doing this by using the inbuilt wetting-and-drying process in Thetis, which follows the approach detailed in Kärnä et al. (2011): the total water depth, $H$, is modified using the expression

$$
\tilde{H}:=\eta-h+f(H),
$$

where $\eta$ is the elevation, $h$ is the bed height, and

$$
f(H):=\frac{1}{2}\left(\sqrt{H^{2}+\delta^{2}}-H\right),
$$

as shown in Figure 3. Here $\delta$ is the wetting-and-drying parameter which Kärnä et al. (2011), recommend is set approximately equal to $d\|\nabla h\|$ where $d$ is the typical length scale of the mesh. Thus, the hydrodynamic equations in our model become

$$
\frac{\partial \tilde{H}}{\partial t}+\nabla \cdot(\tilde{H} \mathbf{U})=0
$$




$$
\frac{\partial \mathbf{U}}{\partial t}+\mathbf{U} \cdot \nabla \mathbf{U}+\mathbf{g} \nabla \eta=v \nabla^{2} \mathbf{U}-\frac{C_{h}(\tilde{H})}{\tilde{H}}\|\mathbf{U}\| \mathbf{U}
$$

where $\mathbf{U}$ is the depth-averaged velocity, $\mathbf{g}$ is gravity, $v$ the viscosity parameter and $C_{h}$ the bed friction. Following Funke et al. (2017), to avoid non-differentiable functions, we make the following smooth approximation to the norm operator in (10)

$$
\|\mathbf{U}\| \approx \sqrt{\|\mathbf{U}\|^{2}+\zeta^{2}}
$$

In Funke et al. (2017), $\zeta$ is set equal to the wetting-and-drying parameter. However, the issue of non-differentiability exists independent of the wetting-and-drying formulation and so here we do not follow this choice. In the test case in Section 4.2, we experimented with values of $\zeta$ between 0.1 and 1 and chose a value of 0.4 because we found this value is large enough for the model not to crash due to non-differentiability issues, but not too large that the friction term spuriously affects the velocity.

To simulate the suspended sediment transport in the fluid, we use an advection-diffusion equation for the sediment concentration. The non-conservative form

$$
\frac{\partial C}{\partial t}+\nabla \cdot\left(F_{\mathrm{corr}} \mathbf{U} C\right)=\varepsilon_{s} \nabla^{2} C+\frac{E_{b}-D_{b}}{H}
$$

is used in Clare et al. (2021b) where $C$ is the depth-averaged sediment concentration, $\varepsilon_{s}$ the diffusivity coefficient, $E_{b}$ the erosion flux, $D_{b}$ the deposition flux, $H$ the depth and $F_{\text {corr }}$ a correction factor which accounts for the fact that depthaveraging the product of two variables is not equivalent to multiplying two depth-averaged variables (see Clare et al. (2021b)). However, the wetting-and-drying scheme used is known to leak sediment. Thus, in order to ensure sediment is conserved when using this wetting-and-drying scheme, in this work we consider the following conservative form,

$$
\frac{\partial}{\partial t}(\tilde{H} C)+\nabla \cdot\left(F_{\text {corr }} \mathbf{U} \tilde{H} C\right)=\varepsilon_{s} \nabla^{2}(\tilde{H} C)+E_{b}-D_{b}
$$

where $\tilde{H}$ is the modified water depth given by (7). Instead of solving for $C$, we solve for the depth-integrated concentration, $\tilde{H} C$, which allows us to use the same finite element formulation that is used for the non-conservative sediment equation. To verify that our conservative scheme has improved the sediment conservation, we consider the simple Thacker test case of oscillations in a paraboloid bowl with diameter $430.620 \mathrm{~m}$ (Thacker, 1981). The hydrodynamic version of this test case is presented in Balzano (1998) and we refer the reader here for more details. The free surface is initially a paraboloid of revolution with a depth of approximately $50 \mathrm{~m}$ which oscillates inside the bowl with no forcing, but does not leave the bowl: the problem is closed hydrodynamically. We introduce a Gaussian blob of sediment in the wet part of the domain defined by the expression

$$
C\left(t_{0}\right)= \begin{cases}100 \exp ^{-\left(x^{2}+y^{2}\right) / 100000} & H \geq 0 \\ 0 & H<0\end{cases}
$$

and run the simulation for $6 \mathrm{~h}$. The principal differences between the conservative and non-conservative schemes are due to the advection term and therefore to avoid unnecessary complications we set the erosion and deposition terms to 0 . At each timestep, $t_{n}$, we calculate the normalised mass error using the following formula

$$
\frac{\int C\left(t_{n}\right) \tilde{H}\left(t_{n}\right) d x-\int C\left(t_{0}\right) \tilde{H}\left(t_{0}\right) d x}{\int C\left(t_{0}\right) \tilde{H}\left(t_{0}\right) d x} .
$$

We find that the normalised mass error is $\mathscr{O}\left(10^{-2}\right)$ for the non-conservative sediment equation (12) but $\mathscr{O}\left(10^{-12}\right)$ for the conservative sediment equation (13), which is close to the numerical precision of the model. Thus, the conservative sediment equation conserves sediment at a much better rate than the non-conservative scheme.

To complete our hydro-morphodynamic model, we use the Exner equation, which governs how the suspended sediment and bedload transport affect the bed. This is unaffected by wetting-and-drying and thus has the same form as that given in Clare et al. (2021b):

$$
\frac{\left(1-p^{\prime}\right)}{m_{f}} \frac{\partial z_{b}}{\partial t}+\nabla \cdot \mathbf{Q}_{b}=D_{b}-E_{b}
$$

where $p^{\prime}$ represents the porosity, $m_{f}$ a morphological scale factor, $z_{b}$ the bathymetry (also known as the bed profile) and $\mathbf{Q}_{b}$ the bedload transport. The morphological scale factor is used to artificially increase the rate of bedlevel changes compared with the underlying hydrodynamics and thus save computational time when simulating long-term morphodynamic change (see Clare et al. (2021b) for more details). 


\subsection{Sediment slide mechanism}

In order to model coastal test cases, we must add one other component: for test cases with complex bathymetries and wet-dry interfaces - like that in Section 4.3 - it is possible for hydro-morphodynamic models to generate physically unrealistic slopes. Hence, we must add a 'sediment slide' mechanism (sometimes called an avalanche mechanism) to our model. The mechanism used is described in Apsley and Stansby (2008) and adds a component $q_{\text {aval }}$ in the direction of the maximum slope, $\widehat{\mathbf{b}}$, to the Exner equation (16) which becomes

$$
\left(1-p^{\prime}\right) \frac{d z_{b}}{d t}+\nabla_{h} \cdot\left(\mathbf{Q}_{\mathbf{b}}+q_{\mathrm{aval}} \widehat{\mathbf{b}}\right)=D_{b}-E_{b}
$$

As we are solving a 2D depth-averaged problem, we ignore the $z$-component of $\widehat{\mathbf{b}}$ and thus

$$
\nabla_{h} \cdot\left(q_{\text {aval }} \widehat{\mathbf{b}}\right)=\nabla_{h} \cdot\left(-\frac{n_{z}^{2} q_{\text {aval }}}{\sin \lambda} \nabla_{h} z_{b}\right)=\nabla_{h} \cdot\left(-\gamma \nabla_{h} z_{b}\right),
$$

which is a diffusion problem. Here $\lambda$ is the slope angle, $n_{z}$ is the $z$-component of the unit normal to the surface, defined by

$$
\widehat{n}_{z}=\frac{1}{\sqrt{1+\left|\nabla_{h} z_{b}\right|^{2}}}
$$

and $q_{\text {aval }}$ is

$$
q_{\text {aval }}= \begin{cases}0.5\left(1-p^{\prime}\right) \zeta^{2} \frac{(\tan \lambda-\tan \phi)}{\cos (\lambda \Delta t)}, & \tan \lambda>\tan \phi, \\ 0, & \text { otherwise },\end{cases}
$$

where $\phi$ is the angle of repose, $p^{\prime}$ the porosity and $\zeta$ the length scale which controls how quickly the sediment is redistributed. In Apsley and Stansby (2008), it is argued that the value of $\zeta$ does not need to be very precise, and thus we set it equal to the approximate mesh step size if the mesh were uniformly distributed. This mechanism stops the slope angle exceeding $\phi$, although it does not model how the sediment slides down the slope.

In order to solve the new Exner equation, we derive the weak form of (18) by multiplying it by the test function, $\psi$, integrating it by parts once and using the divergence theorem to find

$$
-\int_{\Omega} \psi \nabla_{h} \cdot\left(\gamma \nabla_{h} z_{b}\right) d x=\int_{\Omega} \gamma\left(\nabla_{h} \psi\right) \cdot\left(\nabla_{h} z_{b}\right) d x-\int_{\Gamma}\left(\gamma \psi \nabla_{h} z_{b}\right) \cdot \mathbf{n} d S
$$

where $\mathbf{n}$ is the outward pointing normal on each element edge and $\Gamma$ the union of all the element edges. As discussed in Clare et al. (2021b), the Exner equation is solved using a continuous Galerkin finite element discretisation with centred fluxes assumed on the interior edges. This means the values on either side of each interior edge cancel each other out over the whole domain. Furthermore, as we assume Neumann conditions of no flux at the domain boundary, there is no contribution from the exterior edges either so the second term of (21) equals 0 .

In summary, the wetting-and-drying hydro-morphodynamic model with a sediment slide mechanism is given by equations (9), (10), (13) and (16). In both the non-wetting-and-drying and wetting-and-drying cases, the equations are discretised and solved using discontinuous Galerkin finite element methods (see Clare et al. (2021b)). Note that due to the complexity and nonlinearity of this combined system the equations are solved alternately, as opposed to as a monolithic system.

We have thus constructed a full mesh-movement hydro-morphodynamic model framework, where the mesh on which the hydro-morphodynamic model is solved is moved from timestep to timestep using the methods described in Section 2. Note that mesh movement is not constrained to occur at every timestep, meaning we can choose how frequently the mesh is moved. The framework may now be applied to a series of test cases with complex bathymetries and/or wet-dry interfaces.

\section{Test Cases}

\subsection{Complex bathymetry test case: Migrating Trench}

As a first test case, we consider the complex bathymetry case of a migrating trench, based on an experiment in Van Rijn (1980). This test case has already been used in Clare et al. (2021b) for validation of our hydro-morphodynamic model configuration in the case of a fixed uniform mesh

The setup for the migrating trench considered here is the same as that in Clare et al. (2021b), modelling both suspended and bedload transport with magnitude and angle corrections from the slope effect, using the Nikuradse friction formula for $C_{h}$ in (10), and following that work uses a morphological scale factor, $m_{f}$, of 100 to aid computational efficiency. Note that since this test case exists in an entirely wet domain, the wetting and drying scheme is not necessary. Finally, Clare et al. (2021b) shows that this test case is sensitive to the value specified for diffusivity, $\varepsilon_{s}$. Altering 


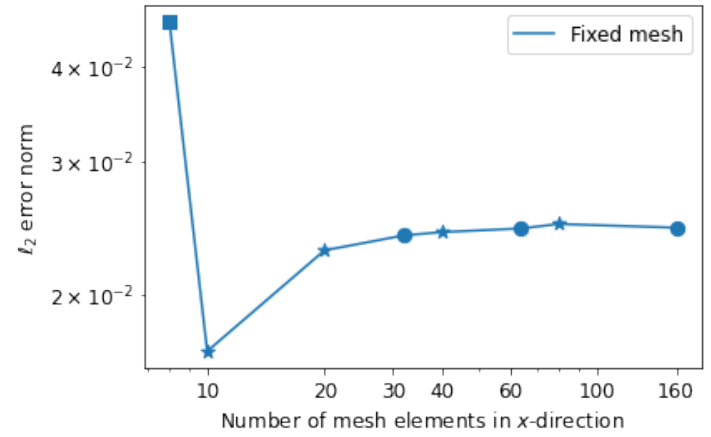

(a) Total error against experimental data

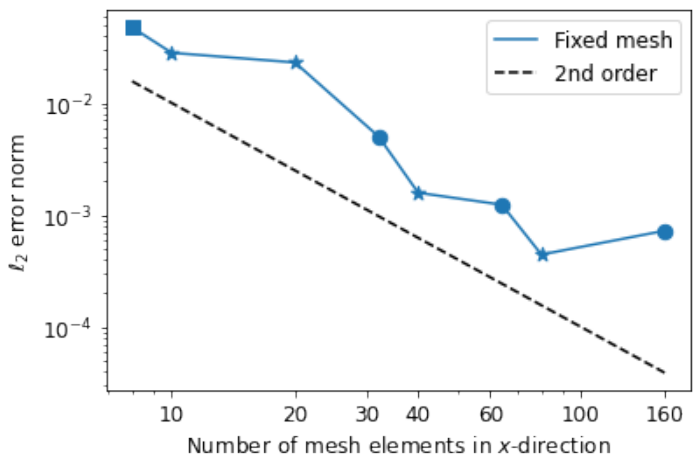

(b) Discretisation error against a reference solution with 320 mesh elements

Fig. 4 Errors in the final bed profile for a series of fixed uniform meshes for the migrating trench test case. The marker shapes distinguish differences in the definition of the initial profile on the mesh: for the circle points the initial trench profile is well-defined on the mesh; for the star and square points the initial trench profile is ill-defined but in different ways

the mesh resolution will change the effective numerical diffusivity of the model, which makes assessing performance against experimental data challenging, due in part to the issue of "getting the right answer for the wrong reasons". Thus we calibrate a value for diffusivity from the experimental data using a uniform fixed mesh with $\Delta x=0.05 \mathrm{~m}$ ( $320 \mathrm{mesh}$ elements in the $x$-direction) in the model. We use a gradient-based optimisation routine to perform optimal parameter estimation and compute the gradients using the adjoint method. More detail on the use of the adjoint framework with the hydro-morphodynamic model can be found in Clare et al. (2021a). We find that the optimal value of the diffusivity coefficient is $\varepsilon_{s}=0.18011$ to five significant figures. Throughout this section, this value is used as the diffusivity parameter.

\subsubsection{Fixed uniform meshes}

Before applying mesh movement methods, we study how the error varies on a series of fixed meshes varying from 320 mesh elements in the $x$-direction down to only 8 elements in the $x$-direction. Throughout this work, the error in the bed profile is calculated at the end of the simulation. The existence of experimental data for this test case means that we can calculate the total error, which is equal to the model error (the error due to using a simplified model to approximate a real world problem), plus discretisation error (the error arising from using a finite mesh to solve the model equations). In this section, the error is calculated using a pointwise $\ell_{2}$ error norm at the location of the data points in the experiment.

Figure 4a shows the total error between the final bed profile from the experimental data and from the model output for a series of fixed uniform meshes. In this figure, the points marked with a star are the results where the number of mesh elements in the $x$-direction $=[10,20,40,80]$ and the points marked with a square are those where the number of mesh elements in the $x$-direction $=8$. This distinction has been made because for both the star and square points the initial profile of the trench is incorrectly defined on meshes but in a different way. For example, instead of the second slope starting at the correct location of $x=9.5 \mathrm{~m}$, for the star points it starts at $x=9.6 \mathrm{~m}$, whereas for the square point it starts at $10 \mathrm{~m}$. These differences affect the results on the fixed uniform mesh. For the remaining circle points (number of mesh elements in the $x$-direction $=[32,64,160,320])$, the initial profile is correctly defined. This difference in the initial trench profile definition results in fluctuations in the error trend. It is surprising that with 10 elements, the total error is lower than that obtained on finer meshes. This is likely due to this test case's sensitivity to diffusivity and the fact that the effective diffusivity is different at the coarser mesh resolutions. Despite these fluctuations, the general trend is that the value of the error norm begins to plateau around 20 mesh elements and by 32 mesh elements the error has clearly converged to a value of approximately $0.0244 \mathrm{~m}$. This is because, once the initial trench profile is well-defined on the mesh, as noted in Clare et al. (2021b), the model is then quite insensitive to changes in $\Delta x$ and $\Delta t$ and thus the total error in the solution is dominated by the model error rather than the discretisation error from the mesh.

To better understand the discretisation error, we calculate the error norm between the final bed profile for different mesh resolutions compared to the final bed profile obtained with the finest fixed uniform mesh with 320 mesh elements in the $x$-direction $(\Delta x=0.05 \mathrm{~m})$. Figure $4 \mathrm{~b}$ shows that as the mesh becomes finer the discretisation error decreases with an order of approximately $(\Delta x)^{2}$. Note, we again separate out visually the number of mesh elements in $x$-direction $=[10,20,40,80]$ marked with a star, the number of mesh elements in $x$-direction $=8$ marked with a square and the remaining marked with a circle. Like with the total error the sets converge at slightly different rates. Comparing Figure $4 \mathrm{a}$ and $4 \mathrm{~b}$ shows that, when there are 20 mesh elements or fewer, the total error is dominated by the discretisation error, but after this point the total error begins to be dominated by the model error. This is because above 20 mesh elements the initial trench profile is either well-defined or close to being well-defined even on a fixed uniform mesh, and so the discretisation error is small. 

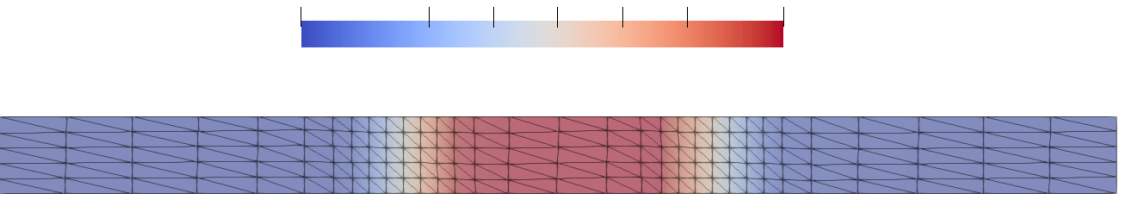

$\mathrm{t}=0 \mathrm{~h}$

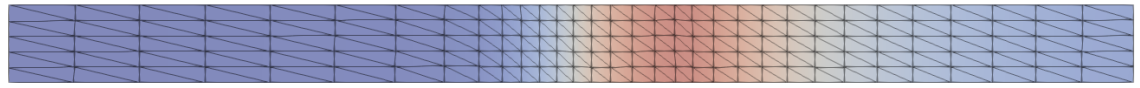

$\mathrm{t}=7.5 \mathrm{~h}$

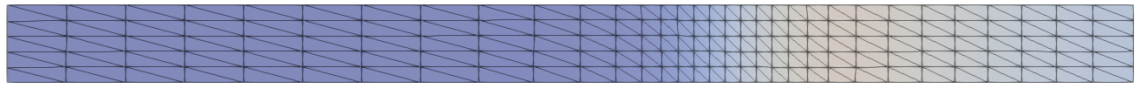

$\mathrm{t}=15 \mathrm{~h}$

Fig. 5 Snapshots of bathymetry and the underlying mesh for a moving mesh simulation of the migrating trench test case. The monitor function (22) has been applied to a mesh with 32 elements in the $x$-direction in the case $\alpha=\beta=3$. Results are shown at three timepoints demonstrating mesh movement to capture bed evolution to the right

\subsubsection{Mesh movement}

In order to apply mesh movement to the test case, we must choose an appropriate monitor function. From studying the fixed uniform meshes, we have determined that the main source of discretisation error in this test case is due to the fact that the initial trench profile is not well-defined on the mesh. Even if a mesh is chosen so the profile is initially well-defined, as soon as the simulation starts, the bed begins to move so the profile may quickly become ill-defined (hence the differences in total error even for the circle points in Figure 4a). Furthermore, there are large sections of the trench profile which are flat and are relatively unchanged during the simulation, especially at the inflow. Therefore, a good choice of monitor function for this test case is one that results in increased mesh resolution in regions where the bed gradient and/or curvature is high and reduced mesh resolution where the bed gradient and/or curvature is lower. We thus consider here the monitor function

$$
m(x, y)=1+\max _{x, y}\left(\alpha \frac{\left\|\underline{H}\left(z_{b}\right)\right\|_{F}}{\max _{x, y}\left\|\underline{H}\left(z_{b}\right)\right\|_{F}}, \beta \frac{\left\|\nabla z_{b}\right\|_{2}}{\max _{x, y}\left\|\nabla z_{b}\right\|_{2}}\right),
$$

where $\underline{H}\left(z_{b}\right)$ represents the Hessian of the bathymetry, $\|\cdot\|_{F}$ the Frobenius norm. This introduces two user-defined parameters which control the effect of the underlying bathymetry on mesh movement; $\alpha$ controls the effect of the second order derivative (curvature), whilst $\beta$ controls the effect of the first-order derivatives (slope). Note that both the Frobenius norm and the 2-norm are taken on an element-by-element basis and then projected into the $\mathbb{P} 1$ space rather than being calculated component-wise. This is because we found that computing the norms component-wise results in an insufficiently smooth monitor function, leading to model divergence. Using an element-wise formulation introduces additional numerical diffusion which helps to counteract this.

Figure 5 shows an example of how the mesh is moved using (22) for this particular test case. Note the mesh hardly moves in the $y$-direction because the bathymetry is uniform in this direction. 


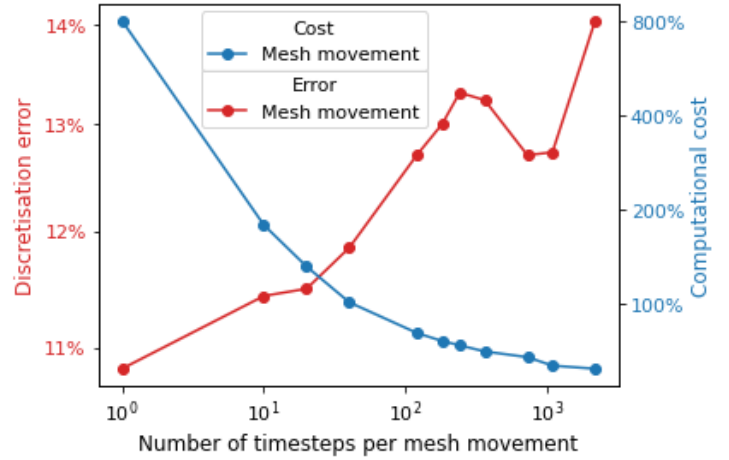

Fig. 6 Trade-off between discretisation error and computational cost due to mesh movement frequency for the migrating trench test case. The monitor function (22) has been applied to a mesh with 32 elements in the $x$-direction in the case $\alpha=\beta=3$. Errors and times are percentages relative to the fixed mesh case

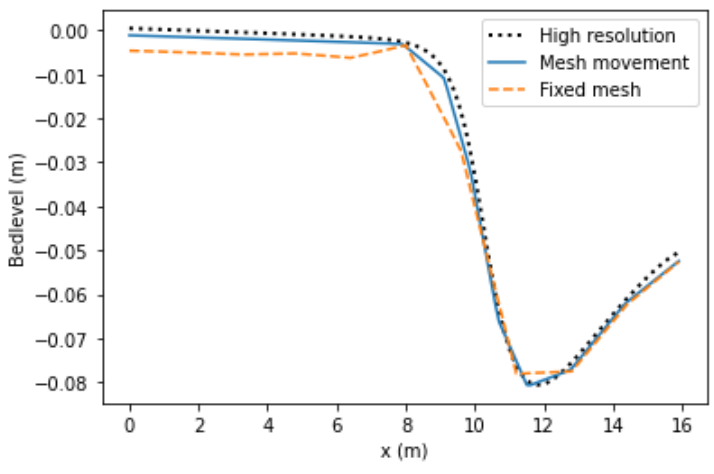

Fig. 7 Comparison of final bedlevels resulting from fixed and moving mesh simulations of the migrating trench test case on a mesh with 32 elements in the $x$-direction. The moving mesh simulation applies the monitor function (22) with $\alpha=\beta=3$ every 40 timesteps. The final bedlevel due to a high resolution fixed mesh simulation is also shown

With the mesh movement method used in this work, we can choose how frequently the mesh is moved. If the modification to the mesh coordinates proposed by (M-A) is only minor then solving this equation at every timestep of our hydro-morphodynamic model may prove an unnecessary expense. Figure 6 shows the effect of changing the frequency of the mesh movement. The more timesteps between each mesh movement, the greater the discretisation error, but the lower the computational cost. This is to be expected as mesh movement methods have a non-negligible computational cost. Note that the total number of timesteps in this simulation is 2160 and therefore in the lowest frequency case the mesh is only moved once during the simulation (at the initial time). Significantly, the figure shows that if a large enough number of timesteps per mesh movement is chosen, we can reduce the computational cost below that of using a fixed mesh and still be more accurate. This supports our argument, at least for this simple test case, that mesh movement can not only reduce error but also reduce the computational cost of the simulation. In the remainder of this section, we choose to move the mesh after every 40 timesteps of the hydro-morphodynamic model (equivalent to moving the mesh 54 times during the simulation), as this provides a good balance between computational cost and accuracy. Figure 7 compares the final bedlevel obtained by moving the mesh at this frequency with the bedlevel obtained using a fixed uniform mesh with the same number of elements, and the 'true' value obtained using a high resolution mesh with 320 mesh elements in the $x$-direction. It shows the mesh movement solution is much more accurate than the fixed mesh solution and thus that only moving the mesh every 40 timesteps is sufficient to see notable improvements.

The mesh movement method used in this work also allows the user to set the values of $\alpha$ and $\beta$ in (22), which control the mesh movement. In order to determine optimum values of these parameters, we conduct a small sensitivity study, but in future work will seek a gradient-based approach (see Section 5). Mesh movement methods have no effect on model error, only on the discretisation error and thus for brevity, we only show the results of the study for discretisation error. Figure 8 shows that for all number of mesh elements, the discretisation error is minimised when $\alpha$ is small (approximately 3). For the smaller number of elements, the discretisation error is significantly smaller than the discretisation error for the fixed mesh $(\alpha=\beta=0)$ and including the first order derivative of the bathymetry in the monitor function is important (i.e. $\beta \neq 0$ ). However, as the number of elements increases the effect of mesh movement on the discretisation error decreases and the first order derivative becomes less important. There is also a clear distinction in the way the discretisation error varies with $\alpha$ and $\beta$ above and below 20 mesh elements. This distinction is also seen in Figure 4 which shows that below 20 mesh elements the discretisation error dominates the model error but above 20 mesh elements the model error dominates the discretisation error. This distinction exists because above 20 mesh elements the initial trench profile is either well-defined or close to being well-defined even on a fixed uniform mesh (which is not the case below 20 mesh elements) and so the discretisation error is already very small $\left(\mathscr{O}\left(10^{-3}\right)\right.$ ).

Thus, we can conclude that for this test case a good general parameter choice for the monitor function (22) is $\alpha=3$ and $\beta=0$ (for large numbers of mesh elements in the $x$-direction) and $\alpha=\beta=3$ (for small numbers of mesh elements in the $x$-direction). The greater dependence on the second order derivative than the first order derivative is predictable because the regions of the trench which are most difficult to capture are the corners where the second derivative is high.

\subsubsection{Introducing a slope in the y-direction}

The test case considered so far in this section is effectively 1D with little variation in the bathymetry or flow in the $y$-direction. However, most hydro-morphodynamic problems are effectively $2 \mathrm{D}$ in nature and thus we modify the test case by introducing a slope of 0.1 in the $y$-direction. All setup parameters are kept the same and the initial profile is shown in Figure 9. 

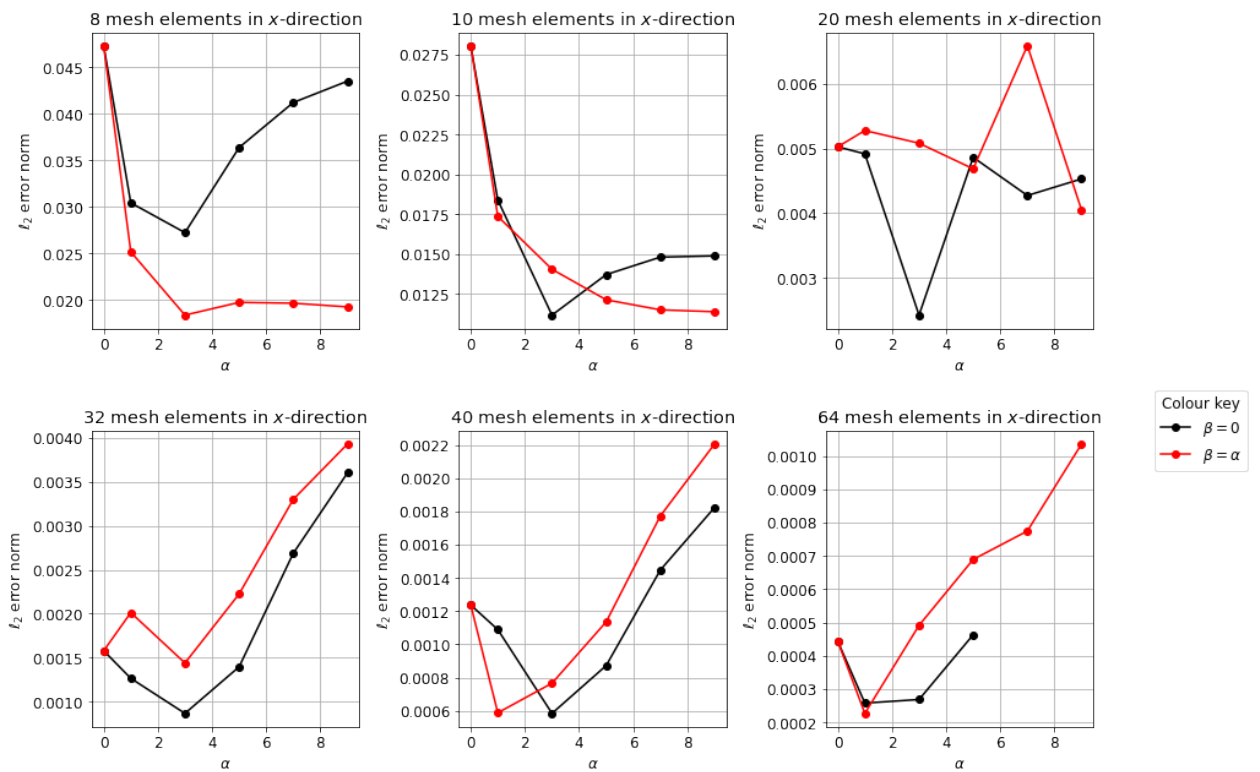

Fig. 8 Discretisation error curves for moving mesh simulations of the migrating trench test case under different values of $\alpha$ and $\beta$ in monitor function (22)

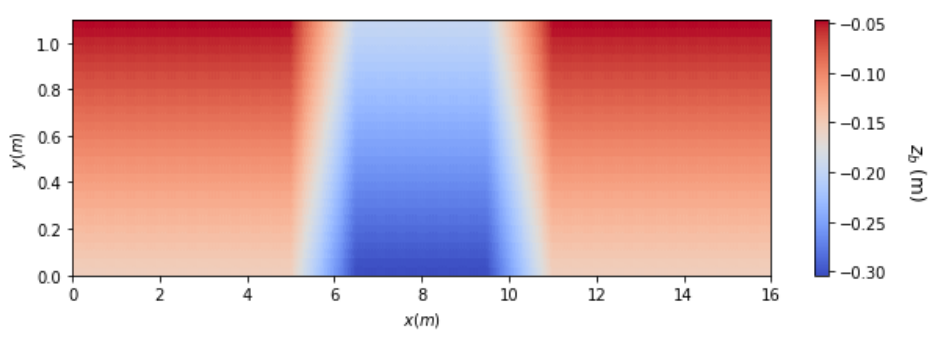

Fig. 9 Initial bed profile for the modified migrating trench test case

The aim of this modified migrating trench test case is to show the generality of our mesh movement framework and that, if the monitor function parameters are known for a similar simple test case, then a full sensitivity analysis is of fixed uniform meshes, in order to compare their accuracy to the accuracy of the mesh movement framework. We choose $8,10,20,32,40$ and 64 mesh elements in the $x$-direction. As the length in the $y$-direction is one sixteenth that in the $x$-direction, we choose the number of mesh elements in the $y$-direction to be one sixteenth of the number in the $x$-direction (rounding up to the nearest integer where necessary). Note that for the eight elements in the $x$-direction we for some mesh movement in the $y$-direction.

With the extra slope in the $y$-direction, we no longer have experimental data to compare against but can still analyse the discretisation error by considering results obtained on a fixed uniform mesh of 320 mesh elements in the $x$-direction $(\Delta x=0.05 \mathrm{~m})$ and 20 mesh elements in the $y$-direction $(\Delta y=0.055 \mathrm{~m})$ as a high resolution approximation of 'the truth'. Furthermore, as we no longer have pointwise experimental values, from this point forward, the error is calculated using the $\mathscr{L}_{2}$ error norm over the whole domain. We note that for fixed uniform meshes as the mesh becomes finer the discretisation error decreases approximately linearly (see Figure 11b later in the section).

Following our analysis from the original migrating trench test case, for this modified case, we move the mesh every 40 timesteps. In addition, as we are testing the generality of our mesh movement framework, instead of conducting a full sensitivity analysis to determine the parameters in the monitor function (22), we use parameters of the same magnitude as those found in the original test case. The introduction of an extra slope though, suggests that a different relationship between $\alpha$ and $\beta$ may be optimal and thus we conduct a small study. Figure 10 shows the results of this study and shows that for small numbers of mesh elements in the $x$-direction the second order derivative of the bathymetry is the most important, then for 32 mesh elements the first and second order derivatives are of equal importance, and finally for more than 32 mesh elements, the first order derivative becomes the most important. This is predictable from the results from the original test case because for the latter, mesh movement results in a large error reduction for small numbers of mesh elements but when the number of elements is large, it has less of an effect. We infer from this that for the modified test case, for small numbers of mesh elements, the discretisation error is dominated by errors in the $x$-direction and thus the 

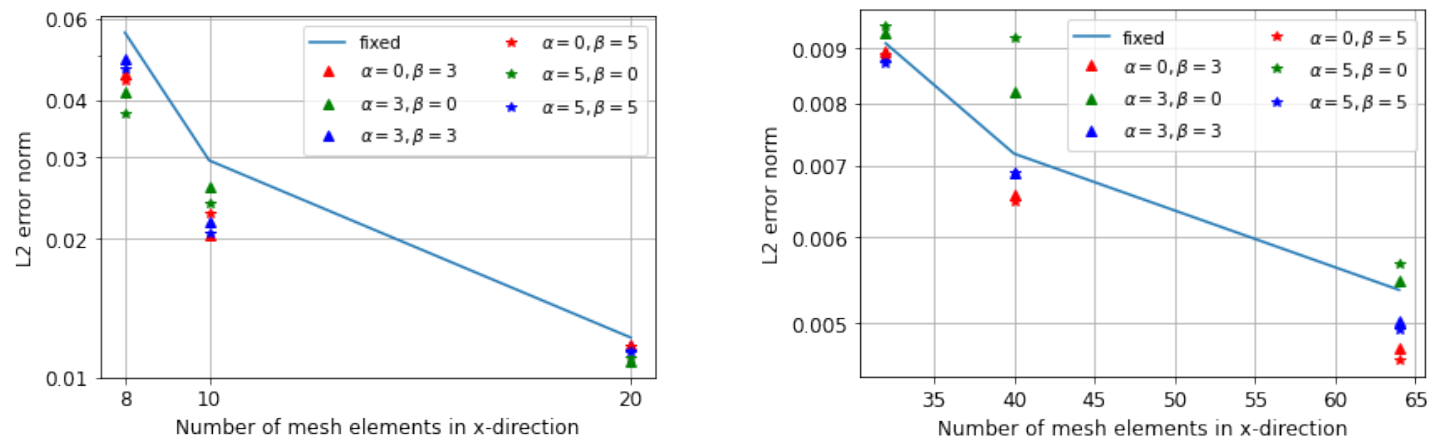

Fig. 10 Discretisation error curves for moving mesh simulations of the modified migrating trench test case under different values of $\alpha$ and $\beta$ in $(22)$

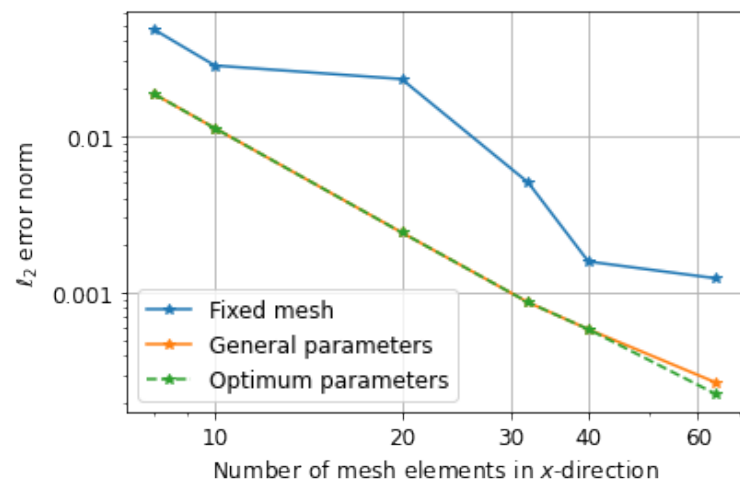

(a) Original (Section 4.1.1 and 4.1.2)

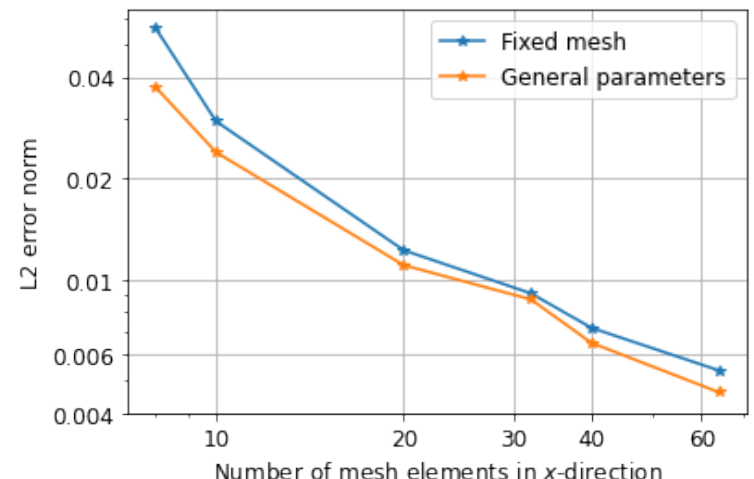

(b) Modified (Section 4.1.3)

Fig. 11 Discretisation error comparison of fixed mesh and moving simulations of the original and modified migrating trench test case. Both general and optimal parameters for the monitor function (22) are considered for the original version. Note that for the original case, the error shown is the pointwise $\ell_{2}$ error norm and for the modified case the error shown is the L 2 error norm over the whole domain

second order derivative is important (as in Section 4.1), but as the number of mesh elements increases the error in the $y$-direction starts to dominate meaning the first order derivative becomes the most important due to the linear slope in the $y$-direction.

Figure 10 also shows that using monitor parameters with a greater magnitude (a magnitude of 5) than that used in Section 4.1 (a magnitude of 3) results in a greater error reduction. This is due to the fact that for the modified case, the bathymetry is more complex and so slightly greater mesh movement is required. This suggests that whilst the magnitude of monitor function parameters obtained from simpler test cases result in good error reduction in more complex problems, it may be a good idea to increase the magnitude slightly.

Finally, Figures 11a and 11b present a summary of our discretisation error results for the original and modified cases respectively. Figure 11a shows, for a given number of mesh elements, the minimum discretisation error achieved in our sensitivity study from using mesh movement (optimum parameter set) and the discretisation error achieved by using mesh movement with our generalised parameter set $(\alpha=3, \beta=3$ for small numbers of mesh elements in the $x$-direction and $\alpha=3, \beta=0$ for large numbers of mesh elements). Figure 11b only shows the discretisation error achieved by a generalised parameter set ( $\alpha=5, \beta=0$ for small numbers of mesh elements; $\alpha=5, \beta=5$ for 32 mesh elements; and $\alpha=0, \beta=5$ for large numbers of mesh elements) because it was not necessary to conduct a full sensitivity analysis for this test case given that we had already done so for the original case. Moving mesh methods consistently result in a lower error than fixed mesh methods and furthermore our general parameter choice produces very similar error results to the optimum values. Thus, we have shown that mesh movement methods can be used to improve accuracy and reduce computational cost for quasi-1D and 2D cases with non-trivial bathymetries. We have also been able to use this test case to draw some conclusions about general good parameter choices for (22). In the next section, we test these general optimum parameter choices on a completely different 2D problem to see if they are again optimal or close to optimal choices. 


\subsection{Wet-dry interface test case: Beach Profile}

We have shown that moving mesh methods can improve accuracy and efficiency for test cases with steep gradients in a fully wet domain. Coastal problems often have a wet-dry interface, for example as a wave or tide moves up a beach. These problems have been historically difficult to solve because of their computational expense, but mesh adaptation methods provide a way to retain accuracy, whilst improving efficiency. Mesh movement methods have been used previously to successfully simulate a wet-dry interface, for example in Zhou et al. (2013)). However, to the best of our knowledge they have not previously been used to solve coupled hydro-morphodynamic cases with wet-dry interfaces.

In this section, we consider the test case of a wave running up a beach slope, whose setup is shown in Figure 12. The incoming wave is governed by

$$
\mathbf{u}=\cos \left(-\frac{1}{2} t\right) \quad \text { and } \quad h=\frac{1}{4} \cos \left(-\frac{1}{2} t\right) \quad \text { at } x=0 \mathrm{~m} .
$$

The total simulated time is $3600 \mathrm{~h}$ (approximately equivalent to 5 months) with a morphological scale factor of 10,000 , meaning the full wave passes over the beach over one hundred times, enough to cause notable erosion and deposition.

For this test case, we use a similar hydrodynamic setup to that used for the Balzano test case in Kärnä et al. (2011). Following that work, instead of using the Nikuradse friction formula as in previous test cases, the Manning friction formula is used to determine the bed friction

$$
C_{h}=g \frac{n^{2}}{H^{1 / 3}},
$$
for sandy coasts. However, whilst in Kärnä et al. (2011) only the hydrodynamics is modelled, here morphodynamic effects are also considered. Both suspended sediment and bedload transport are simulated using the wetting-and-drying conservative form of the hydro-morphodynamic model discussed in Section 3. The magnitude corrections to the slope to account for gravitational effects are also applied (see Clare et al. (2021b) for more details). The parameters used in

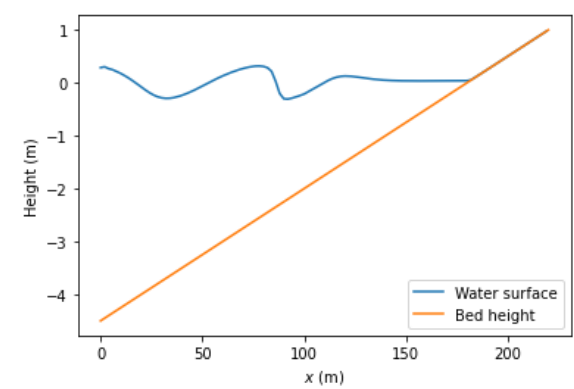

Fig. 12 Initial setup of beach test case

\begin{tabular}{|l|l|}
\hline Variable Name & Variable Value \\
\hline Length in $x$-direction & $220 \mathrm{~m}$ \\
Length in $y$-direction & $10 \mathrm{~m}$ \\
Morphological simulation time & $3600 \mathrm{~h}$ \\
Morphological scale factor & 10000 \\
Median particle size $\left(d_{50}\right)$ & $2 \times 10^{-4} \mathrm{~m}$ \\
Sediment density $\left(\rho_{s}\right)$ & $2650 \mathrm{~kg} \mathrm{~m}^{-3}$ \\
Water density $\left(\rho_{f}\right)$ & $1000 \mathrm{~kg} \mathrm{~m}^{-3}$ \\
Kinematic viscosity $\left(v_{0}\right)$ & $0.5 \mathrm{~m}^{2} \mathrm{~s}^{-1}$ \\
Bed sediment porosity $\left(p^{\prime}\right)$ & 0.4 \\
Diffusivity $\left(\varepsilon_{s}\right)$ & $100 \mathrm{~m}^{2} \mathrm{~s}^{-1}$ \\
Manning friction coefficient $(n)$ & $0.02 \mathrm{sm}^{1 / 3}$ \\
Wetting-and-drying parameter $(\delta)$ & $0.025 \mathrm{~m}^{1 /}$ \\
\hline
\end{tabular}

Table 1 Parameter values used in the beach test case

Note our model does not currently have the ability to simulate shoaling waves and thus we have used a viscosity "sponge" in the dry area to dissipate wave energy, with the viscosity defined by

$$
v(x, y):= \begin{cases}v_{0} & x<100, \\ v_{0}(-399+4 x) & x \geq 100,\end{cases}
$$

where $v_{0}$ is the kinematic viscosity given in Table 1 . Given the test case setup, we can predict that the incoming wave will produce two different types of sediment transport behaviour in the domain. At the input of the domain, we expect large scale erosion as the wave entering the domain acts as a breaking wave would in a normal coastal setup. Further along the domain, we would expect smaller ripple effects to occur as the wave passes over the bed. We could make an educated assumption for where the location of the breaking wave effects ends and the ripple effects starts. However, Figure 13 shows that, even for the coarsest fixed uniform mesh considered in this section (44 mesh elements in the $x$-direction), we can deduce the limit is approximately $x=70 \mathrm{~m}$. To confirm that this limit does not change when the mesh becomes finer, Figure 13 also shows the evolution profile for the finest mesh considered in this section (440 mesh elements in the $x$-direction).

The erosion at the input of the domain clearly dominates the sediment transport behaviour in the domain and any mesh movement will likely focus on this part. Thus, in order to be able to improve the accuracy of the predictions of the ripple like behaviour, throughout this section we also consider the discretisation error over the subdomain separately to the discretisation error over the whole domain. The part the user is more interested in modelling will determine the 


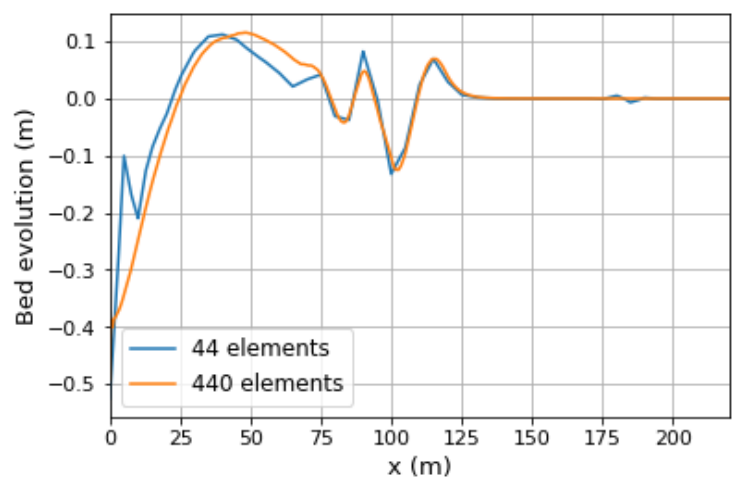

Fig. 13 Total bed evolution in the beach test case over the whole domain after $3600 \mathrm{~h}$. Results due to fixed uniform meshes with 44 and 440 mesh elements are presented

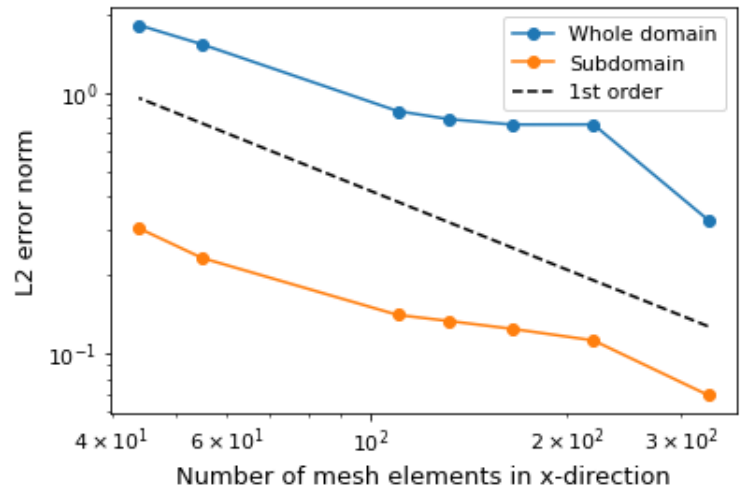

Fig. 14 Discretisation error in a simulation of the beach test case based on a fixed uniform mesh with 44 elements. The reference solution uses a mesh with 440 elements. The absolute $\mathscr{L}_{2}$ errors over both the whole domain and the subdomain are presented

\subsubsection{Fixed uniform meshes}

To begin with, we consider a series of fixed meshes varying from a uniform spacing of 440 mesh elements in the $x$-direction $(\Delta x=0.5 \mathrm{~m})$ to 44 mesh elements $(\Delta x=5 \mathrm{~m})$. In order to keep the elements in the fixed mesh roughly uniform, an appropriate $\Delta y$ is also chosen. Note that we set $\Delta t=0.5 \mathrm{~s}$ for all the simulations in this section, in order to ensure that the wave is correctly defined. Because this is a theoretical test case for which there exists no real data, we use the model solution at 440 mesh elements in the $x$-direction $(\Delta x=0.5 \mathrm{~m})$ and 20 mesh elements in the $y$-direction $(\Delta y=0.5 \mathrm{~m})$ as a high resolution approximation of 'the truth' and are thus showing the estimates of the discretisation error. Note for this section the discretisation error is the $\mathscr{L}_{2}$ error norm over the domain of interest.

Figures 14 shows that, as the mesh becomes finer, the discretisation error decreases approximately linearly over both the whole domain and the subdomain. Already we see a difference in the error between the two domains, with the error converging more uniformly in the subdomain than in the whole domain. Note that the error norms presented are absolute and not relative, and thus, because the subdomain errors are calculated over a smaller domain, they are smaller in magnitude than the whole domain errors.

\subsubsection{Mesh movement}

To apply mesh movement methods, we use the same monitor function as that used in the previous test cases (22) but with a slight modification. For this test case, we are principally interested in bed changes which occur in the direction perpendicular to the shoreline ( $x$-axis) and thus smooth the monitor function in the cross-shore direction ( $y$-direction) by applying Laplacian smoothing,

$$
\frac{m_{\text {smooth }}-m}{\Delta t}=K \frac{\partial^{2}}{\partial y^{2}}\left(m_{\text {smooth }}\right), \text { where } \quad K=N \frac{\Delta x^{2}}{4 \Delta t} .
$$

Here $N$ is equivalent to the number of applications of a $(1,-2,1)$ filter, which we choose to be 40 .

As in the previous sections, we also choose the mesh movement frequency. In previous simulations, we used a frequency of $\mathscr{O}\left(5 \times 10^{2}\right)$ and could reasonably expect again that this would be appropriate. (Note that the frequency must exactly divide the total number of model timesteps, so we cannot use exactly the same value every time.) However, upon running a sensitivity study, Figure 15a shows that, when considering the whole domain, moving the mesh four imes during the simulation (i.e. at least every 648 timesteps) results in a significant reduction in the discretisation error compared to the fixed mesh. This is predictable because we know the error for the whole domain case is focused at the domain input, where the bed evolution pattern remains steady throughout the simulation, i.e. the areas at the input with the greatest erosion rates when the bed begins to evolve are the same areas with the greatest erosion rates at the end of the simulation. Thus, once the mesh movement algorithm has identified the region of interest, it only needs to move the mesh a couple of times in order to optimise the error reduction. It is surprising that if the mesh movement frequency increases from this point, the error increases slightly from 59\% to $59.5 \%$ but potential reasons for this will be discussed in the next paragraph. Note, a frequency of $\mathscr{O}\left(5 \times 10^{2}\right)$ as used in previous test cases would still result in an good error reduction, just not an optimum cost reduction.

If only the subdomain is considered, Figure $15 \mathrm{~b}$ shows that the number of mesh movements during the simulation does affect the discretisation error over this subdomain. Here a frequency of $\mathscr{O}\left(5 \times 10^{2}\right)$ timesteps per mesh movement would be appropriate, and when we run the sensitivity study we see that the discretisation error is marginally more 
minimised by moving the mesh every 72 timesteps, but that every value of this order results in an error value close to the minimum. Surprisingly, moving the mesh more frequently than this increases the error even more significantly than in the whole domain case. This may be because the mesh becomes too concentrated around areas of complex bathymetry, meaning the incoming wave is ill-defined. Alternatively, because of the lack of experimental data, we do not know the true solution for this problem and thus, it may be that with more frequent mesh movement, the model captures an aspect of the problem which is missed by the fixed uniform mesh. Thus, in cases where both the hydrodynamics and morphodynamics change frequently throughout the simulation, this suggests for the optimum accuracy and cost improvement, a slightly larger frequency than that used in the tests cases in Sections 4.1 and 4.1.3 may be more appropriate.

In both the whole domain and subdomain case, the mesh movement frequency may be chosen so that the computational cost is less than the fixed mesh algorithm, whilst still being more accurate. Thus, following our observations, when considering the whole domain error, the mesh is moved every 648 timesteps of the hydro-morphodynamic model and when considering the subdomain error, the mesh is moved every 72 timesteps of the hydro-morphodynamic model.

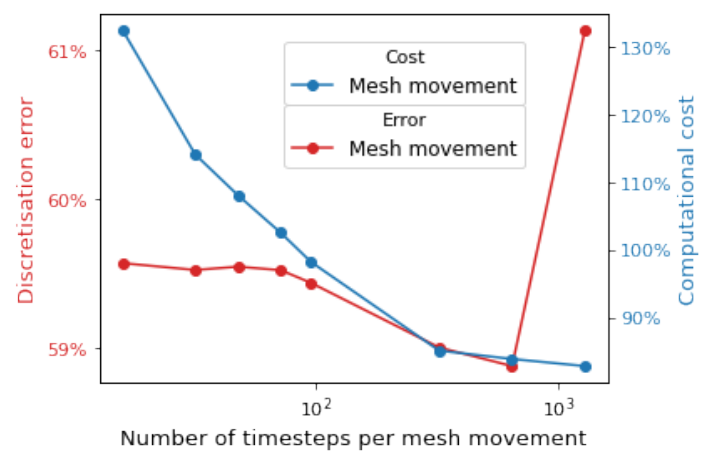

(a) Whole domain, $\alpha=0, \beta=5$

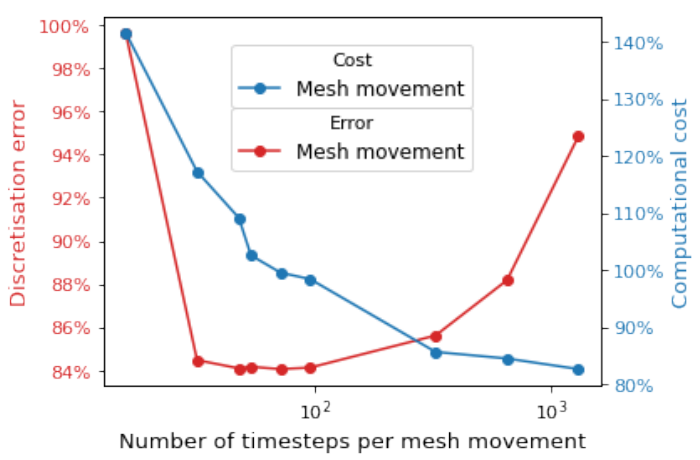

(b) Subdomain, $\alpha=7, \beta=0$

Fig. 15 Trade-off between discretisation error and computational cost due to mesh movement frequency for the beach test case. The monitor function (22) has been applied to a mesh with 110 elements in the $x$-direction. Errors and times are percentages relative to the fixed mesh case

We can also choose an $\alpha$ and $\beta$ in (22) to minimise the discretisation error. In the previous section, we found that a value of $\alpha$ or $\beta \approx 3$ for simple cases and $\alpha$ or $\beta \approx 5$ for more complex cases provides a good general optimisation of the discretisation error. Thus, we conduct our sensitivity study using similar values. First, we consider the discretisation error over the whole domain. Figure 16 shows that whilst a magnitude of 3 does minimise the error, a better general magnitude is 5 , which corresponds to an either optimal or very close to optimal minimisation in the discretisation error over the whole domain. This fits with our physical understanding of the problem because the presence of waves makes the problem more complex thus requiring a stronger mesh movement factor. However, the relation between $\alpha$ and $\beta$ is more important and Figure 16 shows that the discretisation error over the whole domain is minimised when $\beta=\mu$ and $\alpha=0$, (where $\mu$ is used as a pseudo-parameter for magnitude in this section). This corresponds to a monitor function which is dependent on the first derivative of the bed. This is what we would expect from the evolution profile in Figure 13 because most of the error is concentrated at the input where the second derivative is small but the first derivative is large. For these values of $\alpha$ and $\beta$, the discretisation error is significantly lower than the discretisation error for the fixed uniform mesh (equivalent to $\mu=0$ ), for all the mesh resolutions considered.

We next consider the discretisation error over the subdomain, $x>70 \mathrm{~m}$. Figure 17 shows that the discretisation error is in general minimised by $\alpha=\mu, \beta=0$, with $\mu \approx 5$ again corresponding to a large degree of minimisation across all resolutions. The exception is when there are 55 mesh elements, where the error is minimised by $\alpha=0$ and $\beta=\mu$. This may be because for this small number of elements, there is a particular structure that is better represented by the first derivative. Nevertheless, for $\alpha=5$ and $\beta=0$ at all mesh resolutions considered, the discretisation error using the mesh movement method is significantly lower than the discretisation error using the fixed mesh method (equivalent to $\mu=0$ ). Setting $\alpha=\mu$ and $\beta=0$ corresponds to a monitor function which is dependent only on the second-order derivatives of the bed. This is again what we would expect from the subdomain evolution profile in Figure 13, where there are large changes in the second-order derivatives in regions of interest. The difference between the optimum relationship between $\alpha$ and $\beta$ for the whole domain case and the subdomain case shows it is correct to consider them separately.

We have thus shown that a value of $\mu=5$ is a good general parameter for optimising the discretisation error in both the subdomain and the whole domain, which is same order as the value found in Section 4.1 for the more complex modified test case. The relationship between $\alpha$ and $\beta$ has again been shown to be physically intuitive, with $\alpha=0, \beta=\mu$ in the whole domain and $\alpha=\mu, \beta=0$ in the subdomain being shown to be the optimal relationships. Using these general parameters, in Figure 18 we consider the crucial relationship of simulation accuracy versus computational cost. This shows that using moving mesh methods results in greater accuracy for the same computational cost as that of a fixed uniform mesh for all mesh movement frequencies considered (with a slight exception when the number of mesh 

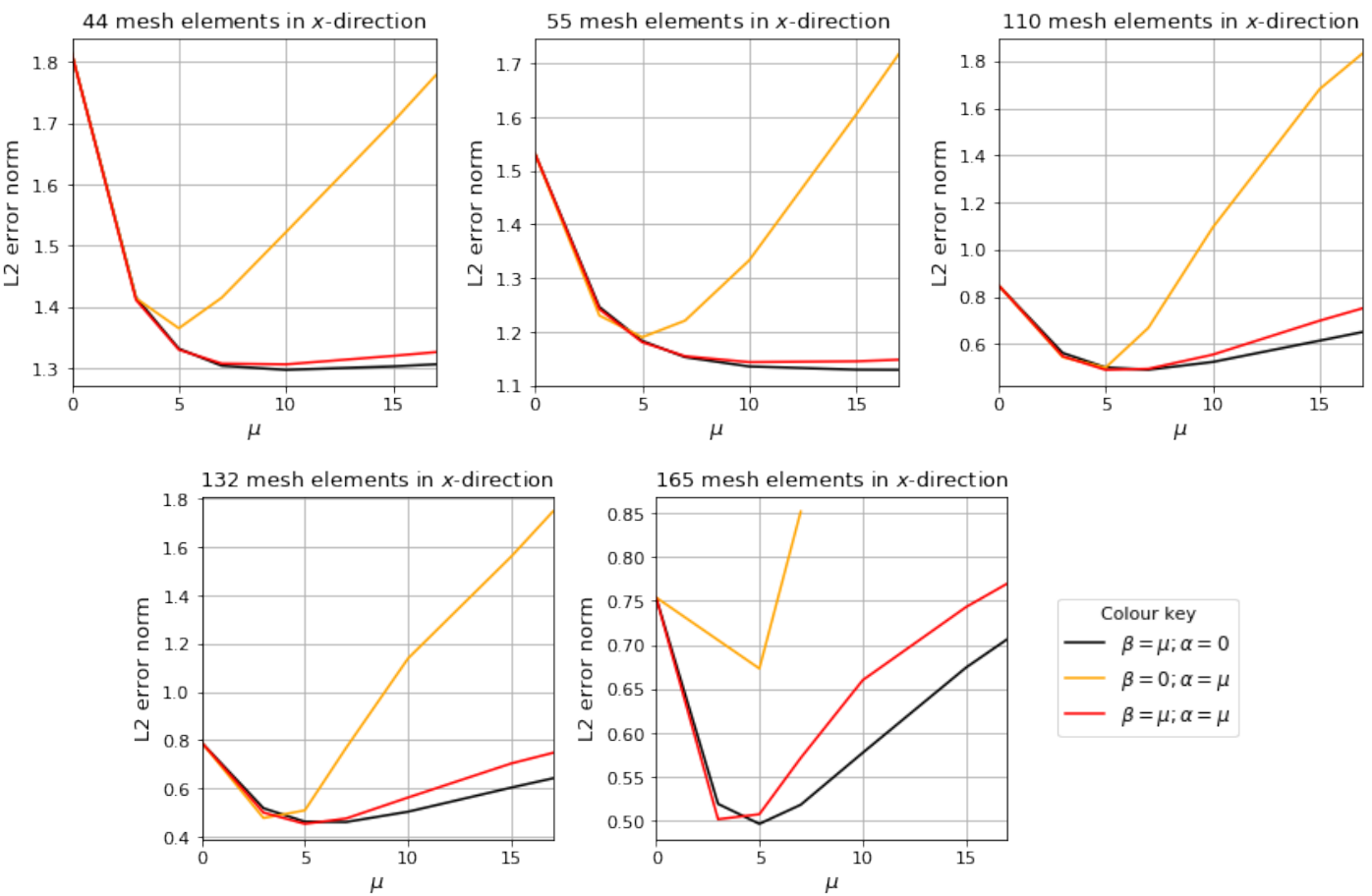

Fig. 16 Discretisation error curves on the whole domain for moving mesh simulations of the beach test case under different values of $\alpha$ and $\beta$ in the monitor function (22)
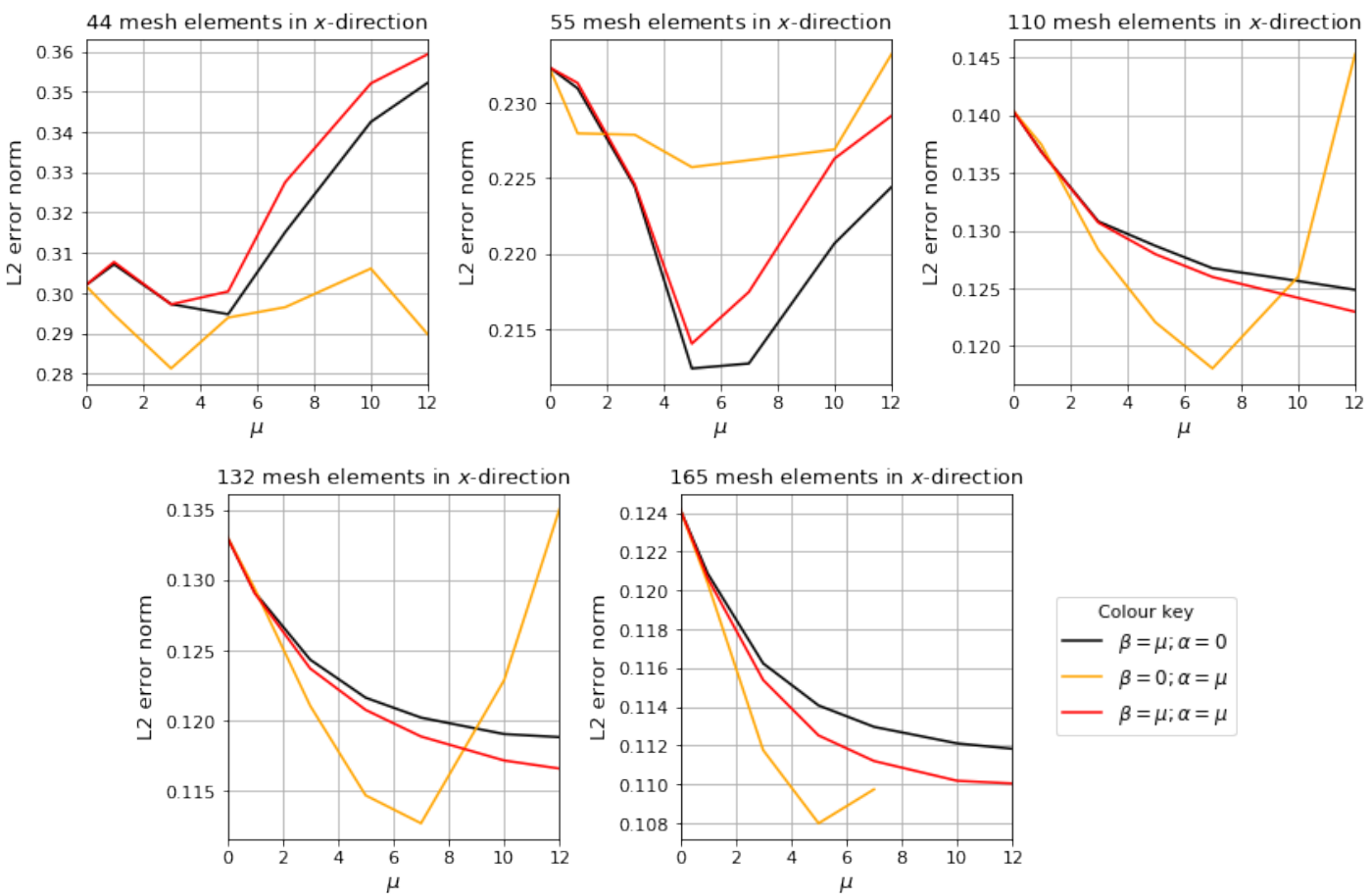

Fig. 17 Discretisation error curves on the subdomain $x>70 \mathrm{~m}$ for moving mesh simulations of the beach test case under different values of $\alpha$ and $\beta$ in the monitor function (22)

elements is low in the subdomain where the accuracy is roughly the same). Figure 18 also shows that as the total cost

of the simulation increases, the cost of each mesh movement increases, too. This is to be expected because the total cost here is a proxy for the number of mesh elements and increasing the number of mesh elements increases the cost of solving (4). Importantly, the proportion of the actual cost of the simulation stays roughly the same and thus using mesh movement methods with high element counts is still computationally efficient when compared to using fixed uniform meshes. 


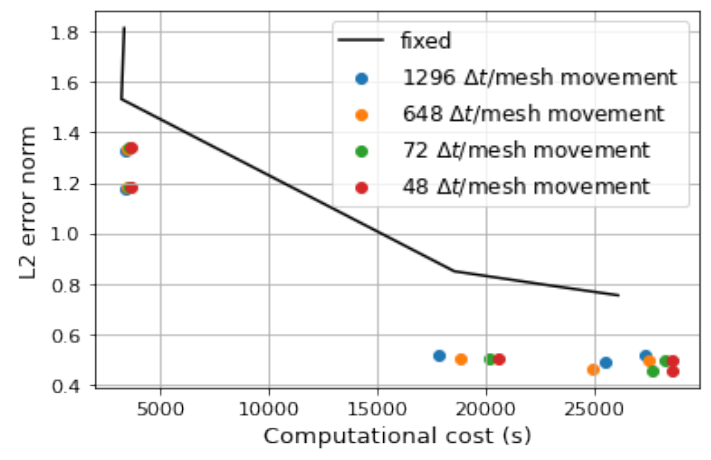

(a) Whole domain

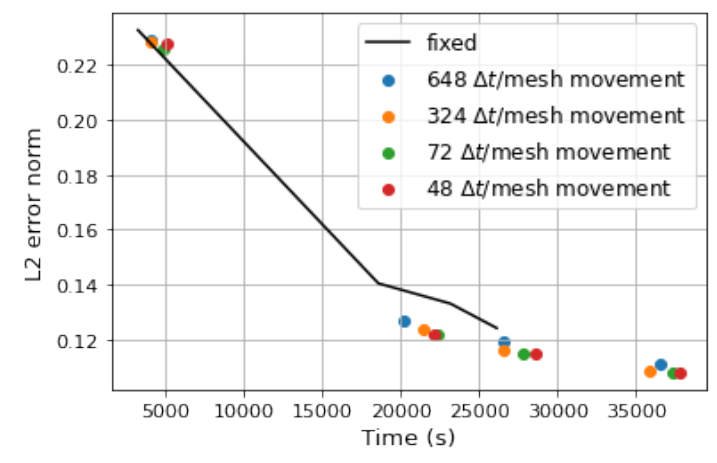

(b) Subdomain

Fig. 18 Computational cost vs discretisation error for fixed mesh and mesh movement methods with general parameters for the beach test case. Note the different points correspond to different numbers of mesh elements and different colours correspond to different mesh movement frequencies

For this relatively complex wetting-and-drying test case, we have thus shown mesh movement methods not only improve accuracy for the same number of mesh elements but can also be used to reduce computational cost, even when using general parameters which have not been tuned for this specific test case.

4.3 Complex bathymetry with a wet-dry interface test case: Tsunami-like wave with an obstacle

As a final test of our mesh movement framework, we consider an example with both a wet-dry interface and a complex initial bathymetry. The setup is a modification of the laboratory experiment in Kobayashi and Lawrence (2004) in which a tsunami-like solitary wave repeatedly breaks over a sloping beach. Inspired by the 2D test case in Hudson and Sweby (2005), which examines how hydro-morphodynamic models deal with obstacles in the bed, we have made the bathymetry more complex by adding a cube obstacle in the wave-approach. The new profile is shown in Figure 19. Recall from Section 3.1 that this more complex bathymetry requires the implementation of the sediment slide mechanism.

Following Kobayashi and Lawrence (2004), we define the incoming wave using the following formula for a positive solitary wave

$$
\eta(t)=H_{\text {wave }} \operatorname{sech}^{2}\left(\sqrt{\frac{3 H_{\text {wave }}}{4 h}} \frac{\sqrt{g\left(H_{\text {wave }}+h\right)}}{h}\left(t-t_{\max }\right)\right)+\eta_{\text {down }},
$$

where $H_{\text {wave }}$ is the average wave height, $h$ the still water depth, $t_{\max }$ the arrival time of the wave crest and $\eta_{\text {down }}$ the initial decrease of the elevation at the beginning of the simulation. In the experiment, the solitary wave is generated 8 times with the bed not adjusted after each wave. For simplicity, we instead generate the wave once and use a morphological acceleration factor of 4 , which is equivalent to generating the wave 4 times. In addition, in the experiment, the simulation is run for $40 \mathrm{~s}$ with $t_{\max }=23.9 \mathrm{~s}$ for each solitary wave, but for the first $20 \mathrm{~s}$ the system is stationary. Thus, we run our model simulation for $20 \mathrm{~s}$ with $t_{\max }=3.9 \mathrm{~s}$ for each solitary wave.

All the parameters used in the simulation are summarised in Table 2 and taken from the experiment in Kobayashi and Lawrence (2004) and also Li and Huang (2013) (which simulated the experiment using Delft3D). Following Li and Huang (2013), we use the Chezy friction formula defined by

$$
C_{\text {chezy }}=\frac{g}{n^{2}}
$$

where $n$ is the Chezy friction parameter. Note that, as in Li and Huang (2013), we do not simulate bedload transport because studies have shown that sediment transport due to tsunami waves mainly occurs due to suspended sediment (Goto et al., 2011). 


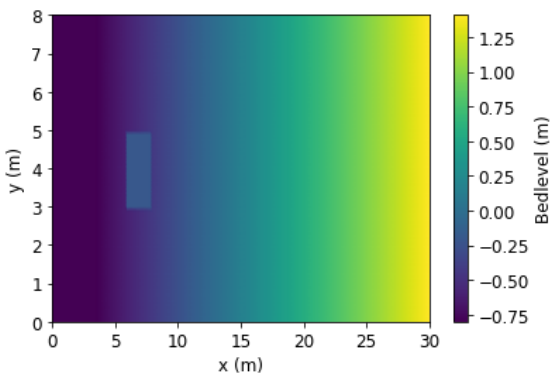

(a) Planar view

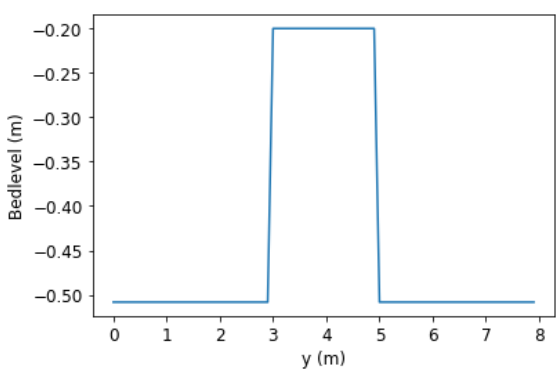

(b) Transect at $x=7 \mathrm{~m}$

\begin{tabular}{|l|l|}
\hline Variable Name & Variable Value \\
\hline Length in $x$-direction & $30 \mathrm{~m}$ \\
Length in $y$-direction & $8 \mathrm{~m}$ \\
Timestep $(d t)$ & $0.025 \mathrm{~s}$ \\
Morphological simulation time & $20 \mathrm{~s}$ \\
Morphological scale factor & 4 \\
Median particle size $\left(d_{50}\right)$ & $1.8 \times 10^{-4} \mathrm{~m}$ \\
Sediment density $\left(\rho_{s}\right)$ & $2650 \mathrm{~kg} \mathrm{~m}^{-3}$ \\
Water density $\left(\rho_{f}\right)$ & $1000 \mathrm{~kg} \mathrm{~m}^{-3}$ \\
Kinematic viscosity $(v)$ & $0.8 \mathrm{~m}^{2} \mathrm{~s}^{-1}$ \\
Bed sediment porosity $\left(p^{\prime}\right)$ & 0.4 \\
Diffusivity $\left(\varepsilon_{s}\right)$ & $1 \mathrm{~m}^{2} \mathrm{~s}^{-1}$ \\
Chezy friction coefficient $(n)$ & $65 \mathrm{~m}^{1 / 2 \mathrm{~s}^{-1}}$ \\
Angle of repose & $20^{\circ}$ \\
Wetting-and-drying parameter $(\delta)$ & $1 / 60 \mathrm{~m}$ \\
Average waveheight $\left(H_{\text {wave }}\right)$ & $0.216 \mathrm{~m}$ \\
Still water depth $(\mathrm{h})$ & $0.18 \mathrm{~m}$ \\
Initial elevation decrease $\left(\eta_{\text {down }}\right)$ & $-0.0025 \mathrm{~m}$ \\
Wave arrival time $\left(t_{\text {max }}\right)$ & $3.9 \mathrm{~s}$ \\
\hline
\end{tabular}

Table 2 Parameter values used in the tsunami with obstacle test case

Fig. 19 Initial bed profile for the tsunami with obstacle test case

\subsubsection{Fixed uniform meshes}

As with the other test cases, we begin by considering a series of fixed meshes with 30, 60, 90, 120 and 150 mesh elements in the $x$-direction corresponding to $8,16,24,32,40$ mesh elements in the $y$-direction respectively, meaning the mesh elements are roughly uniform. Because we have combined two test cases to construct this test case, we no longer have experimental data available. Thus, we use the model solution at 600 mesh elements in the $x$-direction $(\Delta x=0.05 \mathrm{~m})$ and 160 mesh elements in the $y$-direction $(\Delta y=0.05 \mathrm{~m})$ as a high resolution approximation of 'the truth'. The discretisation error in this section is the $\mathscr{L}_{2}$ error over the whole domain.

When we run our hydro-morphodynamic model on these fixed uniform meshes, we find that with only 30 mesh elements in the $x$-direction, our hydro-morphodynamic model crashes no matter how small a timestep is used. This is because at such a coarse resolution, the model cannot accurately simulate the movement of the tsunami-like wave along the slope and instead unphysical shocks form which cannot be properly resolved. For the other fixed uniform meshes, the discretisation error decreases approximately linearly, as the number of mesh elements increases.

\subsubsection{Mesh movement}

Due to the more complex nature of this test case, instead of using the same monitor function as in previous test cases (22), we add a component which tracks the wet-dry interface so the new monitor function is

$$
m(x, y)=1+\mu\left(\max _{x, y}\left(\alpha \frac{\left\|\underline{H}\left(z_{b}\right)\right\|_{F}}{\max _{x, y}\left\|\underline{H}\left(z_{b}\right)\right\|_{F}}, \beta \frac{\left\|\nabla z_{b}\right\|_{2}}{\max _{x, y}\left\|\nabla z_{b}\right\|_{2}}\right)+\frac{\lambda}{\cosh \left(b_{\lambda}\left(\eta-z_{b}\right)\right)^{2}}\right),
$$

where $\eta$ is the elevation, $z_{b}$ the bed level, $b_{\lambda}$ controls the width of the wet-dry interface tracker and $\alpha, \beta$ and $\lambda$ are all user-defined parameters. Note, we set $b_{\lambda}$ equal to 1 for all numbers of mesh elements, apart from for the smallest number of mesh elements when we set $b_{\lambda}$ equal to 5 to ensure that, despite the small number of elements, the wet-dry tracker still has an effect. Figure 20 shows an example of how the mesh moves using this monitor function with 120 mesh elements in the $x$-direction. To better show the mesh movement and bed evolution for this test case with this number of mesh elements, the figure shows the mesh only and the 3D view of the bathymetry separately. The first and second order bathymetry derivatives in (29) cause the mesh to deform around the edges of the block whilst the wet-dry interface monitor tracks the movement of the wave up and down the slope. To illustrate this tracking movement, the wet-dry interface is plotted as a thick black line on Figure 20.

As with the previous test cases, we can choose how frequently the mesh is moved. Figure 21 shows that increasing the frequency of mesh movement decreases the discretisation error and increases the computational cost. Whilst the cost of using mesh movement is always greater than the cost of using a fixed uniform mesh, mesh movement methods 

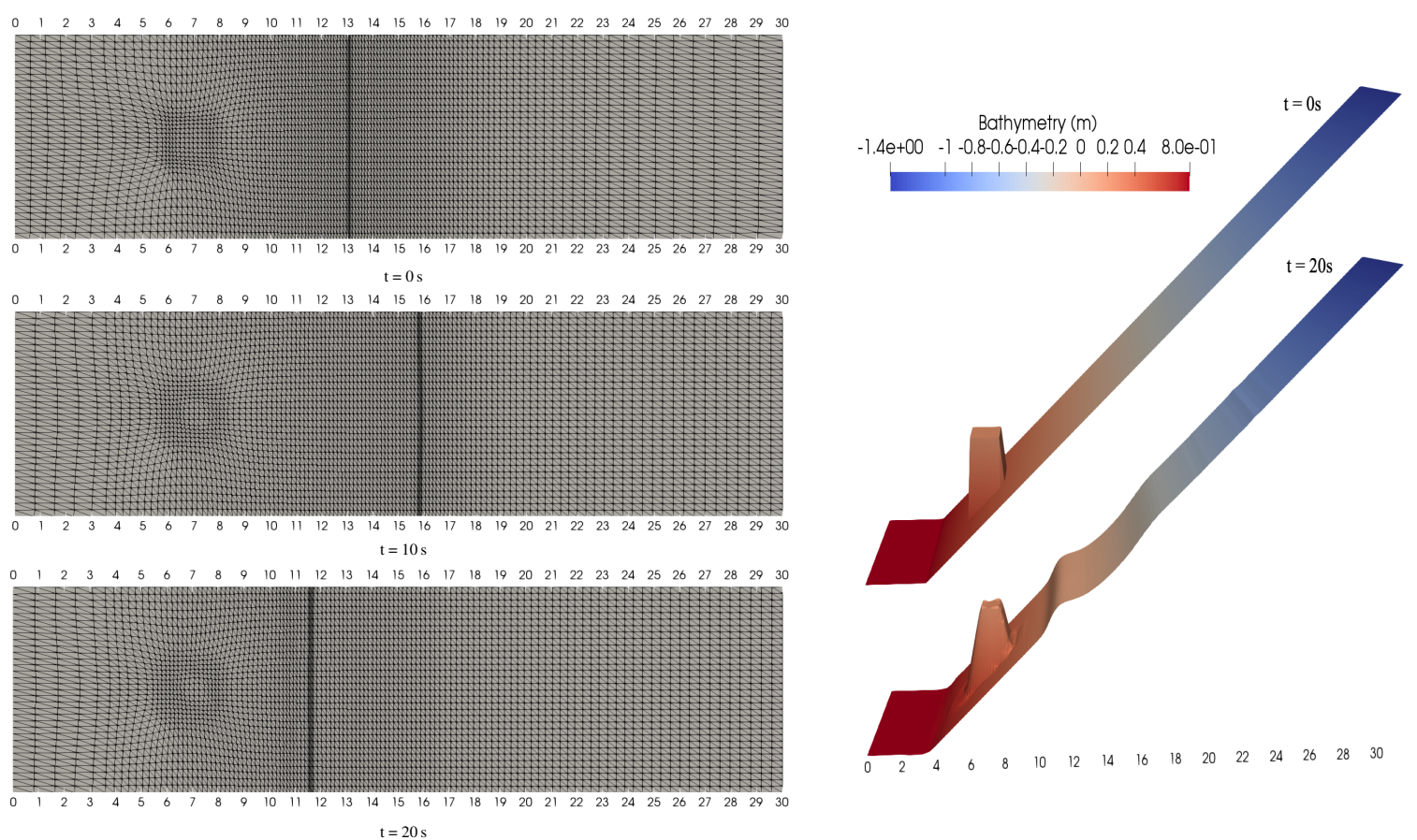

Fig. 20 Mesh movement using (29) for the tsunami with obstacle test case with 120 mesh elements in the $x$-direction and $\mu=15$ and $\alpha=\beta=\lambda=1$ (i.e. an equal contribution from the first and second order bathymetry derivatives and the wet-dry interface tracker). Results are shown with the mesh only at three timepoints with the wet-dry interface shown as a thick black line (LEFT) and in 3D form at two timepoints (RIGHT) demonstrating mesh movement to capture bed evolution to the right

are always at least twice as accurate as the fixed uniform mesh. The accuracy improvement seems to plateau at around 20 timesteps per mesh movement and therefore in the remainder of this section, this is the frequency with which we move the mesh. As the simulation time is $20 \mathrm{~s}$ and the timestep is $d t=0.025 \mathrm{~s}$, this is equivalent to moving the mesh 40 times during the simulation. Figure 22 compares the final bedlevel obtained by moving the mesh at this frequency to the bedlevel obtained using a fixed uniform mesh with the same number of elements and the 'true' value obtained using a high resolution mesh with 600 elements in the $x$-direction. It shows the mesh movement solution is much more accurate than the fixed mesh solution and thus that moving the mesh at this frequency is appropriate for this new more complex monitor function (29).

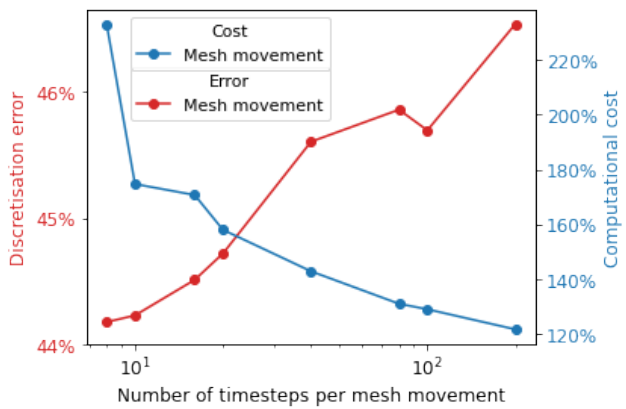

Fig. 21 Trade-off between discretisation error and computational cost due to mesh movement frequency for the tsunami with obstacle test case. The monitor function (29) has been applied to a mesh with 60 elements in the $x$-direction in the case $\mu=7, \alpha=0, \beta=\lambda=1$. Errors and times are percentages relative to the fixed mesh case

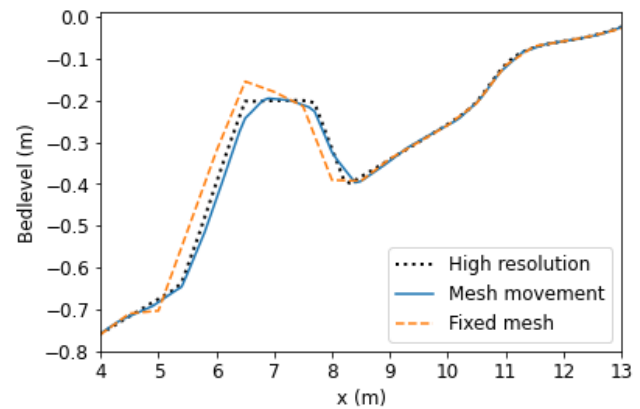

Fig. 22 Comparison of final bedlevels resulting from fixed and moving mesh simulations of the tsunami with obstacle test case on a mesh with 60 elements in the $x$-direction. The moving mesh simulation applies the monitor function (29) with $\mu=7, \alpha=0$, $\beta=\lambda=1$ every 40 timesteps. The final bedlevel due to a high resolution simulation on a fixed mesh with 600 elements in the $x$-direction is also shown

We can also choose $\alpha, \beta$ and $\lambda$ to minimise the discretisation error. In previous test cases in this work, we find that a value of $\alpha$ or $\beta \approx 5$ provides a good general optimisation of the discretisation error and thus we conduct our sensitivity study using similar values. Figure 23 shows the results of this sensitivity study and shows that again a magnitude of 5 provides a good general optimisation of the discretisation error. In all cases, the error is reduced by using mesh 

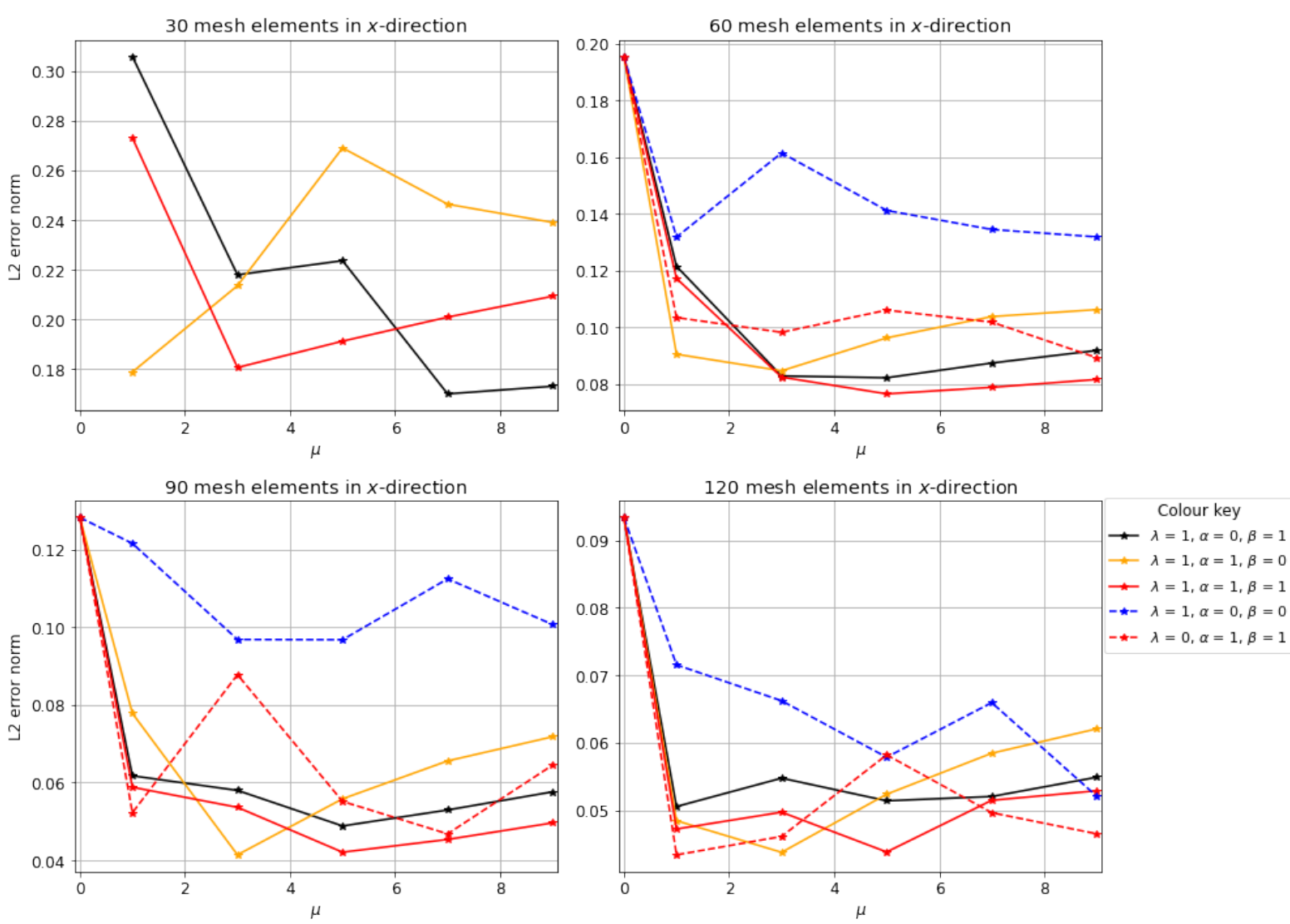

Fig. 23 Discretisation error curves for moving mesh simulations of the tsunami with obstacle test case under different values of $\alpha, \beta$ and $\lambda$ in the monitor function (29)

movement methods compared to the fixed uniform mesh $(\mu=0)$. Note that for 30 mesh element in the $x$-direction, the fixed uniform mesh model crashes which is why no error is plotted for $\mu=0$ on this subfigure.

The figure also shows the effect on the discretisation error of different relationships between $\alpha, \beta$ and $\lambda$ in the monitor function (29). The largest mesh movement errors occur almost always when the monitor function only includes the wet-dry interface tracker $(\lambda=1, \alpha=\beta=0)$. This is understandable given that with this monitor function, the obstacle in the wave-approach is not well-captured. However, in almost all cases the inclusion of the wet-dry interface tracker with some combination of the first and second order derivative of the bathymetry results in a decrease in the discretisation error relative to the tracker not being present. In fact, it is only with the inclusion of the wet-dry interface tracker that our model can properly resolve the wave movement on the coarse mesh with only 30 elements in the $x$-direction. This highlights that appropriate mesh movement can not only decrease computational cost and improve accuracy, but also improve model stability and justifies the use of the more complex monitor function (29) in this test case. A good general choice for the relationship between $\alpha, \beta$ and $\lambda$ is $\alpha=\beta=\lambda=1$, i.e. an equal weighting between the first and second order derivatives of the bathymetry and the wet-dry interface tracker. This makes physical sense because the bathymetry derivatives are necessary to capture the obstacle correctly and the interface tracker is necessary to capture the erosion and deposition caused by the incoming wave. Using this general parameter choice, we can more than double the accuracy of our model for the same number of mesh elements when compared to a fixed uniform mesh, which is a notable result.

We have thus shown that a good general parameter choice for this test case is $\mu=5$ and $\alpha=\beta=\gamma=1$, which is the same magnitude as the complex test cases considered in Section 4.1.3 and 4.2 and the same order of magnitude as the simple test case in Section 4.1. Using these general parameters, in Figure 24 we consider the crucial relationship of simulation accuracy versus computational cost. The figure shows that using mesh movement methods results in both a significant improvement in accuracy and a significant reduction in computational cost, even when general parameters are used rather than optimal parameters. (Note the optimal parameters plotted here are the parameters which provide the smallest error and are not necessarily the fastest simulations, hence why the general parameters perform better in the cost-to-accuracy ratio than the 'optimal' parameters). In many cases, it is possible to halve the discretisation error for the same computational cost, a notable improvement. This is a particularly good result if we wish to assess the uncertainty of this test case, as this requires the model to be run multiple times. In addition, using mesh movement methods reduces the 


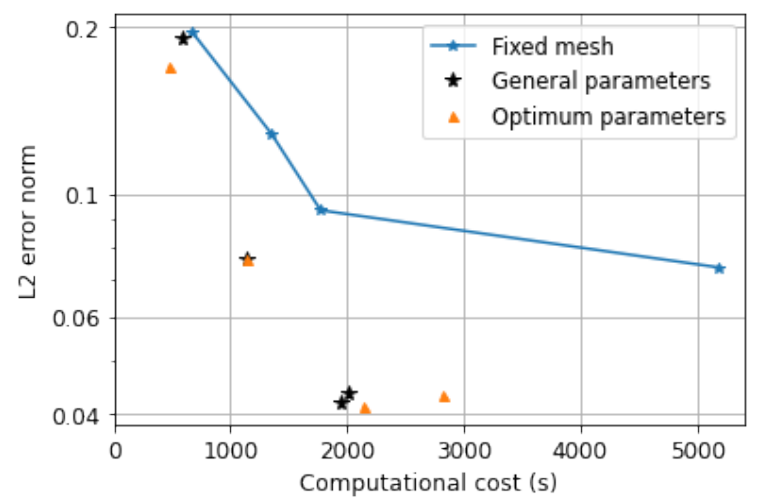

Fig. 24 Computational cost vs discretisation error for fixed mesh and mesh movement methods with general and optimal parameters for the tsunami with obstacle test case. Note the different points correspond to different numbers of mesh elements

number of mesh elements to required to achieve a good accuracy, which means that the memory costs of our simulation are reduced when using adjoint methods through pyadjoint (see Clare et al. (2021a)).

Therefore, we have shown that for test cases with relatively complex bathymetries and a wet-dry interface, we can not only in many cases more than double the accuracy for the same number of mesh elements but also reduce computational cost and improve model stability, even when using general parameters which have not been tuned for this specific test case, a noteworthy result.

\section{Conclusion}

In this work we have implemented a mesh movement scheme as part of a hydro-morphodynamic model for the first time. We have shown that these mesh movement methods can be used to improve accuracy and decrease the computational cost of hydro-morphodynamic test cases with complex bathymetries and/or moving wet-dry interfaces. Moreover, in certain cases we have demonstrated that mesh movement can also improve model stability. A highlight is that these improvements are particularly significant with test cases with wet-dry interfaces, which many coastal zone applications include. This reduction in computational cost, in future work, will allow us to better assess uncertainty since more simulations can be performed for the same cost.

For the mesh movement method considered, we present a monitor function for which the scaling factor that optimises the error reduction is fairly predictable from the physical characteristics of the test case. This will facilitate using this monitor function on further problems. In this work, we have used small scale sensitivity studies to obtain these optimum mesh parameters. In future work, we will seek to use the adjoint framework within Firedrake to allow us to more rigorously determine the optimum values for the scaling parameters. Section 4.1 showed that our hydro-morphodynamic model can be combined with the adjoint framework to determine an optimum diffusivity coefficient, and thus this is a promising line for follow-up research.

\section{Declarations}

\section{Funding}

We acknowledge funding from the Engineering and Physical Sciences Research Council (EPSRC) under grants EP/R512540/1, EP/L016613/1, EP/R007470/1 and EP/R029423/1 and from the Natural Environment Research Council (NERC) under grants NE/M013693/1.

\section{Conflicts of interest}

We confirm that there are no relevant financial or non-financial competing interests to report

\section{Code availability}

The relevant code for the moving mesh hydro-morphodynamic model presented in this work has been stored at at 


\section{References}

Amoudry LO, Souza AJ (2011) Deterministic coastal morphological and sediment transport modeling: A review and discussion. Reviews of Geophysics 49(2)

Apsley DD, Stansby PK (2008) Bed-load sediment transport on large slopes: model formulation and implementation within a RANS solver. Journal of Hydraulic Engineering 134(10):1440-1451

Avdis A, Candy AS, Hill J, Kramer SC, Piggott MD (2018) Efficient unstructured mesh generation for marine renewable energy applications. Renewable Energy 116:842-856

Awanou G (2015) Quadratic mixed finite element approximations of the Monge-Ampère equation in 2d. Calcolo 52(4):503-518

Balzano A (1998) Evaluation of methods for numerical simulation of wetting and drying in shallow water flow models. Coastal Engineering 34(1-2):83-107, DOI 10.1016/S0378-3839(98)00015-5

Barral N, Knepley MG, Lange M, Piggott MD, Gorman GJ (2016) Anisotropic mesh adaptation in Firedrake with PETSc DMPlex. arXiv preprint arXiv:161009874

Benkhaldoun F, El Mahi I, Sari S, Seaid M (2013) An unstructured finite volume method for coupled models of suspended sediment and bed-load transport in shallow water flows. International journal for numerical methods in fluids 72(9):967-993, DOI 10.1002/d.3771

Budd CJ, Williams J (2009) Moving mesh generation using the parabolic Monge-Ampère equation. SIAM Journal on Scientific Computing 31(5):3438-3465

Budd CJ, Huang W, Russell RD (2009) Adaptivity with moving grids. Acta Numerica pp 1-131, DOI 10.1017/ S0962492906400015

Budd CJ, Cullen MJP, Walsh EJ (2013) Monge-Ampére based moving mesh methods for numerical weather prediction, with applications to the eady problem. Journal of Computational Physics 236:247-270

Callaghan DP, Ranasinghe R, Roelvink D (2013) Probabilistic estimation of storm erosion using analytical, semiempirical, and process based storm erosion models. Coastal Engineering 82:64-75

575 Clare MCA, Kramer SC, Cotter CJ, Piggott MD (2021a) Calibration, inversion and sensitivity analysis for hydromorphodynamic models through the application of adjoint methods, in preparation

Clare MCA, Percival JR, Angeloudis A, Cotter CJ, Piggott MD (2021b) Hydro-morphodynamics 2D modelling using a discontinuous Galerkin discretisation. Computers \& Geosciences 146

Dawson C, Sun S, Wheeler MF (2004) Compatible algorithms for coupled flow and transport. Computer Methods in Applied Mechanics and Engineering 193(23-26):2565-2580

Delandmeter P (2017) Discontinuous Galerkin finite element modelling of geophysical and environmental flows. PhD thesis, Universite catholique de Louvain

Donea J, Huerta A, Ponthot JP, Rodríguez-Ferran A (2017) Arbitrary Lagrangian-Eulerian methods. Encyclopedia of Computational Mechanics Second Edition pp 1-23

Farhat C, Degand C, Koobus B, Lesoinne M (1998) Torsional springs for two-dimensional dynamic unstructured fluid meshes. Computer methods in applied mechanics and engineering 163(1-4):231-245

Farrell PE, Ham DA, Funke SW, Rognes ME (2013) Automated derivation of the adjoint of high-level transient finite element programs. SIAM Journal on Scientific Computing 35(4):C369-C393

Funke S, Farrell P, Piggott M (2017) Reconstructing wave profiles from inundation data. Computer Methods in Applied Mechanics and Engineering 322:167-186

Geology Cafe (2015) Chapter 11 - rivers, streams, and water underground. http://geologycafe.com/class/ chapter11.html, accessed on 2020-09-11

Goto K, Takahashi J, Oie T, Imamura F (2011) Remarkable bathymetric change in the nearshore zone by the 2004 Indian Ocean tsunami: Kirinda Harbor, Sri Lanka. Geomorphology 127(1-2):107-116

595 Harris DL, Rovere A, Casella E, Power H, Canavesio R, Collin A, Pomeroy A, Webster JM, Parravicini V (2018) Coral reef structural complexity provides important coastal protection from waves under rising sea levels. Science Advances $4(2)$

Huang W, Ren Y, Russell RD (1994) Moving mesh partial differential equations (MMPDEs) based on the equidistribution principle. SIAM Journal on Numerical Analysis 31(3):709-730

Hudson J, Sweby PK (2005) A high-resolution scheme for the equations governing 2D bed-load sediment transport. International Journal for Numerical Methods in Fluids 47(10-11):1085-1091

Kärnä T, de Brye B, Gourgue O, Lambrechts J, Comblen R, Legat V, Deleersnijder E (2011) A fully implicit wetting-drying method for DG-FEM shallow water models, with an application to the Scheldt Estuary. Computer Methods in Applied Mechanics and Engineering 200(5-8):509-524

Kärnä T, Kramer SC, Mitchell L, Ham DA, Piggott MD, Baptista AM (2017) Thetis coastal ocean model: discontinuous Galerkin discretization for the three-dimensional hydrostatic equations. arXiv preprint arXiv:171108552

Kobayashi N, Lawrence AR (2004) Cross-shore sediment transport under breaking solitary waves. Journal of Geophysical Research: Oceans 109(3), DOI 10.1029/2003jc002084

Lakkis O, Pryer T (2013) A finite element method for nonlinear elliptic problems. SIAM Journal on Scientific Computing 35(4):A2025-A2045 
Lange M, Mitchell L, Knepley MG, Gorman GJ (2016) Efficient mesh management in Firedrake using PETSc-DMPlex. SIAM Journal on Scientific Computing 38(5):S143-S155, DOI 10.1137/15M1026092, 1506.07749

Li L, Huang Z (2013) Modeling the change of beach profile under tsunami waves: A comparison of selected sediment transport models. Journal of Earthquake and Tsunami 7(1), DOI 10.1142/S1793431113500012

Maddison JR, Panourgias I, Farrell PE (2017) libsupermesh version 1.0. Tech. rep., University of Edinburgh

Mayne DA, Usmani AS, Crapper M (2002) An Adaptive Finite Element Solution for Cohesive Sediment Transport. In: Proceedings in Marine Science, vol 5, Elsevier, pp 627-641, DOI 10.1016/S1568-2692(02)80044-4

McManus TM, Percival JR, Yeager BA, Barral N, Gorman GJ, Piggott MD (2017) Moving mesh methods in Fluidity and Firedrake. Tech. Rep. July, Imperial College London, DOI 10.13140/RG.2.2.27670.24648

McRae ATT, Cotter CJ, Budd CJ (2018) Optimal-transport-based mesh adaptivity on the plane and sphere using finite elements. SIAM Journal on Scientific Computing 40(2):1121-1148, DOI 10.1137/16M1109515

Papanicolaou ATN, Elhakeem M, Krallis G, Prakash S, Edinger J (2008) Sediment transport modeling review - current and future developments. Journal of Hydraulic Engineering 134(1):1-14

Piggott MD, Pain CC, Gorman GJ, Power PW, Goddard AJH (2006) $h, r$, and $h r$ adaptivity with applications in numerical ocean modelling. Ocean Modelling 10(1-2 SPEC. ISS.):95-113, DOI 10.1016/j.ocemod.2004.07.007

Piggott MD, Gorman GJ, Pain CC, Allison PA, Candy AS, Martin BT, Wells MR (2008a) A new computational framework for multi-scale ocean modelling based on adapting unstructured meshes. International Journal for Numerical Methods in Fluids 56(8):1003-1015

Piggott MD, Pain CC, Gorman GJ, Marshall DP, Killworth PD (2008b) Unstructured Adaptive Meshes for Ocean Modeling. In: Hecht MW, Hasumi H (eds) Ocean Modeling in an Eddying Regime, American Geophysical Union, pp 383-408

Piggott MD, Farrell PE, Wilson CR, Gorman GJ, Pain CC (2009) Anisotropic mesh adaptivity for multi-scale ocean modelling. Philosophical Transactions of the Royal Society A: Mathematical, Physical and Engineering Sciences 367(1907):4591-4611, DOI 10.1098/rsta.2009.0155, https://royalsocietypublishing.org/doi/pdf/ 10.1098/rsta.2009.0155

Rathgeber F, Ham DA, Mitchell L, Lange M, Luporini F, McRae AT, Bercea GT, Markall GR, Kelly PH (2016) Firedrake: automating the finite element method by composing abstractions. ACM Transactions on Mathematical Software (TOMS) 43(3):1-27

Thacker WC (1981) Some exact solutions to the nonlinear shallow-water wave equations. Journal of Fluid Mechanics 107:499-508

Van Rijn LC (1980) Storm surge barrier Oosterschelde-computation of siltation in dredged trenches: Semi-empirical model for the flow in dredged trenches. Deltares, Delft, The Netherlands

Villaret C, Kopmann R, Wyncoll D, Riehme J, Merkel U, Naumann U (2016) First-order uncertainty analysis using Algorithmic Differentiation of morphodynamic models. Computers \& geosciences 90:144-151

Wallwork JG, Barral N, Ham DA, Piggott MD (2020a) Anisotropic goal-oriented mesh adaptation in Firedrake. In: 28th Intl Meshing Roundtable, Zenodo, DOI 10.5281/zenodo.3653101

Wallwork JG, Barral N, Kramer SC, Ham DA, Piggott MD (2020b) Goal-oriented error estimation and mesh adaptation for shallow water modelling. Springer Nature Applied Sciences 2:1053-1063, DOI 10.1007/s42452-020-2745-9

Weller H, Browne P, Budd C, Cullen M (2016) Mesh adaptation on the sphere using optimal transport and the numerical solution of a Monge-Ampère type equation. Journal of Computational Physics 308:102-123

White AB Jr (1979) On selection of equidistributing meshes for two-point boundary-value problems. SIAM Journal on Numerical Analysis 16(3):472-502

Zhou F, Chen G, Huang Y, Yang JZ, Feng H (2013) An adaptive moving finite volume scheme for modeling flood inundation over dry and complex topography. Water Resources Research 49(4):1914-1928, DOI 10.1002/wrcr.20179 